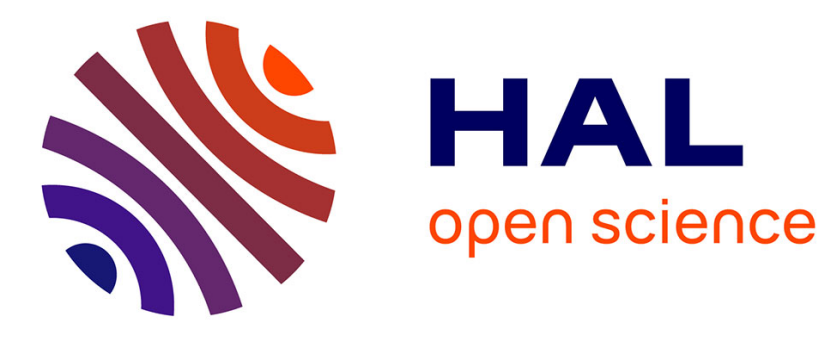

\title{
The Generalized Franchetta Conjecture for Some Hyperkähler Fourfolds
}

Robert Laterveer

\section{To cite this version:}

Robert Laterveer. The Generalized Franchetta Conjecture for Some Hyperkähler Fourfolds. Publications of the Research Institute for Mathematical Sciences, 2019, 55 (4), pp.859-893. 10.4171/PRIMS/55-4-8 . hal-02336792

\section{HAL Id: hal-02336792 \\ https://hal.science/hal-02336792}

Submitted on 29 Oct 2019

HAL is a multi-disciplinary open access archive for the deposit and dissemination of scientific research documents, whether they are published or not. The documents may come from teaching and research institutions in France or abroad, or from public or private research centers.
L'archive ouverte pluridisciplinaire HAL, est destinée au dépôt et à la diffusion de documents scientifiques de niveau recherche, publiés ou non, émanant des établissements d'enseignement et de recherche français ou étrangers, des laboratoires publics ou privés. 


\title{
THE GENERALIZED FRANCHETTA CONJECTURE FOR SOME HYPERKÄHLER FOURFOLDS
}

\author{
ROBERT LATERVEER
}

\begin{abstract}
We obtain a "generalized Franchetta conjecture" type of statement for the Hilbert squares of low genus $K 3$ surfaces, and for the Fano varieties of lines on certain cubic fourfolds.
\end{abstract}

\section{INTRODUCTION}

For a smooth projective variety $X$ over $\mathbb{C}$, let $A^{i}(X):=C H^{i}(X)_{\mathbb{Q}}$ denote the Chow groups (i.e. the groups of codimension $i$ algebraic cycles on $X$ with $\mathbb{Q}$-coefficients, modulo rational equivalence).

The world of Chow groups is still shrouded in mystery, its map containing vast underdeveloped regions only partially sketched in by conjectures [7], [21], [22], [23], [30], [45], [31]. One region on this map that holds particular interest is that of hyperkähler varieties (i.e. projective irreducible holomorphic symplectic manifolds [2], [1]). Here, recent years have seen significant progress in the understanding of Chow groups. Much of this progress centers around the following conjecture:

Conjecture 1.1 (Beauville, Voisin [5], [42]). Let $X$ be a hyperkähler variety. Let $D^{*}(X) \subset$ $A^{*}(X)$ denote the $\mathbb{Q}-$ subalgebra generated by divisors and Chern classes of $X$. Then the cycle class maps induce injections

$$
D^{i}(X) \hookrightarrow H^{2 i}(X, \mathbb{Q}) \quad \forall i
$$

(cf. [5], [42], [4], [8], [35], [10] for cases where conjecture 1.1 is satisfied.)

The "motivation" underlying conjecture 1.1 is that for a hyperkähler variety $X$, the Chow ring $A^{*}(X)$ is expected to have a bigrading $A_{[*]}^{*}(X)$, where the piece $A_{[j]}^{i}(X)$ corresponds to the graded $\mathrm{Gr}_{F}^{j} A^{i}(X)$ for the conjectural Bloch-Beilinson filtration. In particular, it is expected that the subring $A_{[0]}^{*}(X)$ injects into cohomology, and that $D^{*}(X) \subset A_{[0]}^{*}(X)$.

In addition to divisors and Chern classes, what other cycles should be in the subring $A_{[0]}^{*}(X)$ (assuming this subring exists) ? The following conjecture of Voisin provides more candidate members:

2010 Mathematics Subject Classification. Primary 14C15, 14C25, 14C30.

Key words and phrases. Algebraic cycles, Chow groups, motives, Bloch-Beilinson filtration, hyperkähler variety, Lagrangian subvariety, Fano variety of lines on cubic fourfold, $K 3$ surface, Beauville's splitting property, multiplicative Chow-Künneth decomposition, spread of algebraic cycles.

Author address. Institut de Recherche Mathématique Avancée, CNRS - Université de Strasbourg, 7 Rue René Descartes, 67084 Strasbourg CEDEX, FRANCE. robert.laterveer@math.unistra.fr.

Communicated by S. Mukai. Received February 15, 2017. Revised September 17, 2018. 
Conjecture 1.2 (Voisin [47]). Let $X$ be a hyperkähler variety of dimension $n=2 m$. Let $Z \subset X$ be a codimension $i$ subvariety swept out by $i$-dimensional constant cycle subvarieties. There exists a subring $A_{[0]}^{*}(X) \subset A^{*}(X)$ injecting into cohomology, and

$$
Z \in A_{[0]}^{i}(X) .
$$

A constant cycle subvariety is by definition a closed subvariety $T \subset X$ such that the image of the natural map $A_{0}(T) \rightarrow A^{n}(X)$ has dimension 1 . In particular, conjecture 1.2 stipulates that Lagrangian constant cycle subvarieties (i.e., constant cycle subvarieties of dimension $m$ ) should lie in $A_{[0]}^{m}(X)$.

Another conjecture concerns more generally m-dimensional subvarieties $Z \subset X$ that are Lagrangian, i.e. such that

$$
\cup Z: \quad H^{2,0}(X) \rightarrow H^{m+2, m}(X)
$$

is the zero map. Since $H^{*, 0}(X)$ is generated by $H^{2,0}(X)$, we have that

$$
\cup Z: \quad H^{j, 0}(X) \rightarrow H^{m+j, m}(X)
$$

is the zero map for all $j>0$. Since conjecturally, the piece $A_{[j]}^{j}(X)$ is determined by $H^{j, 0}(X)$, and the piece $A_{[j]}^{2 m}(X)$ is determined by $H^{2 m, 2 m-j}(X)$, we arrive at the following conjecture:

Conjecture 1.3. Let $X$ be a hyperkähler variety of dimension $2 m$. Let $Z \subset X$ be a codimension $m$ subvariety that is Lagrangian. Then the map

$$
A_{[j]}^{j}(X) \stackrel{\cdot Z}{\rightarrow} A^{m+j}(X) \stackrel{\cdot b}{\rightarrow} A^{2 m}(X) \rightarrow A_{[j]}^{2 m}(X)
$$

is zero for all $j>0$ and all $b \in A^{m-j}(X)$. (Here, the last arrow is projection to the piece $A_{[j]}^{2 m}(X)$.)

The goal of this note is to provide some examples where conjecture 1.2 and 1.3 are satisfied, by looking at Hilbert squares of $K 3$ surfaces. Here, thanks to the Fourier decomposition of Shen-Vial [38], the Chow groups of $X$ split in a finite number of pieces $A_{(*)}^{*}(X){ }^{1}$

The main result of this note is a "generalized Franchetta conjecture" type of statement for certain Hilbert squares $X$. The statement is that codimension 2 cycles that can be defined relatively live in the subring $A_{(0)}^{*}(X)$ :

Theorem (=theorem 3.1). Let $\mathcal{S} \rightarrow B$ denote the universal family of $K 3$ surfaces of genus $g \in\{2,3,4,5,6,8\}$. Let $\mathcal{X}=\mathcal{S}^{[2]} \rightarrow B$ denote the universal family of Hilbert squares of genus $g$ K3 surfaces (so a fibre $X_{b}$ is the Hilbert square of a genus $g$ K3 surface). Let $\Gamma \in A^{2}(\mathcal{X})$ be a relative cycle. Then

$$
\left.\Gamma\right|_{X_{b}} \in A_{(0)}^{2}\left(X_{b}\right) \quad \forall b \in B .
$$

(Here $A_{(0)}^{2}()$ refers to the Fourier decomposition of Shen-Vial [38].)

\footnotetext{
${ }^{1} \mathrm{NB}$ : we will use the notation $A_{(*)}^{*}()$ for the bigrading that is constructed unconditionally in [38] for certain hyperkähler fourfolds. The notation $A_{[*]}^{*}()$, that occurs only in this introduction, refers to a conjectural bigrading with the property that $A_{[j]}^{i}(X)$ is related to the graded $\mathrm{Gr}_{F}^{j} A^{i}(X)$ for the conjectural Bloch-Beilinson filtration. Hence, the (unconditionally existing) bigrading $A_{(*)}^{*}()$ is a candidate for the (only ideally existing) bigrading $A_{[*]}^{*}()$.
} 
A second result addresses conjecture 1.3:

Theorem (=theorem 3.2). Let $\mathcal{X} \rightarrow B$ be as in theorem 3.1. Let $\Gamma \subset \mathcal{X}$ be a codimension 2 subvariety. Assume the restriction

$$
\Gamma_{b}:=\left.\Gamma\right|_{X_{b}} \quad \in A^{2}\left(X_{b}\right)
$$

is Lagrangian (i.e., $\cup \Gamma_{b}: H^{2,0}\left(X_{b}\right) \rightarrow H^{4,2}\left(X_{b}\right)$ is the zero map), for very general $b \in B$. Then

$$
\cdot \Gamma_{b}: \quad A_{h o m}^{2}\left(X_{b}\right) \rightarrow A^{4}\left(X_{b}\right)
$$

is the zero map, for all $b \in B$.

Section 4 presents some concrete applications of these results. Here is one of them:

Corollary (=corollary 4.1). Let $S$ be a general $K 3$ surface of genus 5 , and let $X=S^{[2]}$ be the Hilbert square. Let $A \subset X$ be a general fibre of the Lagrangian fibration $\phi: X \rightarrow \mathbb{P}^{2}$ of proposition 2.25. Let $b \in A^{4}(X)$ be a 0 -cycle of the form

$$
b=A \cdot a+p \quad \in A^{4}(X),
$$

where $a \in A^{2}(X)$ and $p$ is a sum of intersections of divisors and Chern classes of $X$. Then $b$ is rationally trivial if and only if $b$ is of degree 0.

The proof of theorems 3.1 and 3.2 is based on Voisin's method of "spread" of algebraic cycles [43], [44], [45], [46], combined with results of Pavic-Shen-Yin on the generalized Franchetta conjecture for $K 3$ surfaces [33].

It would be interesting to extend theorem 3.1 to Hilbert squares of $K 3$ surfaces of higher genus, and also to other hyperkähler varieties. ${ }^{2}$ For starters, we prove this for Fano varieties of cubic fourfolds containing two planes (theorem 5.1).

Conventions. In this article, the word variety will refer to a reduced irreducible scheme of finite type over $\mathbb{C}$. A subvariety is a (possibly reducible) reduced subscheme which is equidimensional.

All Chow groups will be with rational coefficients: we will denote by $A_{j}(X)$ the Chow group of $j$-dimensional cycles on $X$ with $\mathbb{Q}$-coefficients; for $X$ smooth of dimension $n$ the notations $A_{j}(X)$ and $A^{n-j}(X)$ are used interchangeably.

The notations $A_{\text {hom }}^{j}(X), A_{A J}^{j}(X)$ will be used to indicate the subgroups of homologically trivial, resp. Abel-Jacobi trivial cycles. For a morphism $f: X \rightarrow Y$, we will write $\Gamma_{f} \in$ $A_{*}(X \times Y)$ for the graph of $f$. The contravariant category of Chow motives (i.e., pure motives with respect to rational equivalence as in [37], [31]) will be denoted $\mathcal{M}_{\text {rat }}$.

We will use $H^{j}(X)$ to indicate singular cohomology $H^{j}(X, \mathbb{Q})$.

\section{PRELiminaries}

\subsection{The Fourier decomposition.}

\footnotetext{
${ }^{2}$ Results of this kind can be found in [12], which was written after the present paper.
} 
Theorem 2.1 (Shen-Vial [38]). Let $S$ be a $K 3$ surface, and let $X=S^{[2]}$ be the Hilbert scheme of length 2 subschemes of $S$. There is a decomposition

$$
A^{i}(X)=\bigoplus_{\substack{0 \leq j \leq i \\ j \text { even }}} A_{(j)}^{i}(X),
$$

with the following properties:

(i) $A_{(*)}^{*}(X)$ is a bigraded ring;

(ii) $A_{(j)}^{i}(X) \subset A_{\text {hom }}^{i}(X)$ for $j>0$.

Proof. This is essentially [38, Theorem 2], combined with the fact that there is a class $L \in$ $A^{2}(X \times X)$ lifting the Beauville-Bogomolov class and satisfying certain equalities, which is [38, Part 2].

Theorem 2.2 (Shen-Vial [38]). Let $Y \subset \mathbb{P}^{5}(\mathbb{C})$ be a smooth cubic fourfold, and let $X=F(Y)$ be the Fano variety of lines in $Y$. There is a decomposition

$$
A^{i}(X)=\bigoplus_{\substack{0 \leq j \leq i \\ j \text { even }}} A_{(j)}^{i}(X),
$$

with the following properties:

(i) $A_{(j)}^{i}(X) \subset A_{\text {hom }}^{i}(X)$ for $j>0$;

(ii) if $Y$ is very general, $A_{(*)}^{*}(X)$ is a bigraded ring.

Proof. This follows again from [38, Theorem 2]. Point (ii) is [38, Theorem 3].

\subsection{MCK decomposition.}

Definition 2.3 (Murre [30]). Let $X$ be a smooth projective variety of dimension $n$. We say that $X$ has a CK decomposition if there exists a decomposition of the diagonal

$$
\Delta_{X}=\pi_{0}+\pi_{1}+\cdots+\pi_{2 n} \text { in } A^{n}(X \times X),
$$

such that the $\pi_{i}$ are mutually orthogonal idempotents in $A^{n}(X \times X)$ and $\left(\pi_{i}\right)_{*} H^{*}(X)=H^{i}(X)$.

(NB: "CK decomposition" is shorthand for "Chow-Künneth decomposition".)

Remark 2.4. The existence of a CK decomposition for any smooth projective variety is part of Murre's conjectures [30], [21], [22].

Definition 2.5 (Shen-Vial [38]). Let $X$ be a smooth projective variety of dimension $n$. Let $\Delta_{X}^{s m} \in A^{2 n}(X \times X \times X)$ be the class of the small diagonal

$$
\Delta_{X}^{s m}:=\{(x, x, x) \mid x \in X\} \subset X \times X \times X .
$$

An MCK decomposition is a CK decomposition $\left\{\pi_{i}^{X}\right\}$ of $X$ that is multiplicative, i.e. it satisfies

$$
\pi_{k}^{X} \circ \Delta_{X}^{s m} \circ\left(\pi_{i}^{X} \times \pi_{j}^{X}\right)=0 \text { in } A^{2 n}(X \times X \times X) \text { for all } i+j \neq k .
$$

(NB: "MCK decomposition" is shorthand for "multiplicative Chow-Künneth decomposition".) $A$ weak MCK decomposition is a CK decomposition $\left\{\pi_{i}^{X}\right\}$ of $X$ that satisfies

$$
\left(\pi_{k}^{X} \circ \Delta_{X}^{s m} \circ\left(\pi_{i}^{X} \times \pi_{j}^{X}\right)\right) *(a \times b)=0 \text { for all } a, b \in A^{*}(X) \text { and for all } i+j \neq k \text {. }
$$


Remark 2.6. The small diagonal (seen as a correspondence from $X \times X$ to $X$ ) induces the multiplication morphism

$$
\Delta_{X}^{s m}: \quad h(X) \otimes h(X) \rightarrow h(X) \text { in } \mathcal{M}_{\text {rat }} .
$$

Suppose $X$ has a CK decomposition

$$
h(X)=\bigoplus_{i=0}^{2 n} h^{i}(X) \text { in } \mathcal{M}_{\text {rat }} .
$$

By definition, this decomposition is multiplicative if for any $i, j$ the composition

$$
h^{i}(X) \otimes h^{j}(X) \rightarrow h(X) \otimes h(X) \stackrel{\Delta_{X}^{s m}}{\longrightarrow} h(X) \text { in } \mathcal{M}_{\text {rat }}
$$

factors through $h^{i+j}(X)$.

If $X$ has a weak MCK decomposition, then setting

$$
A_{(j)}^{i}(X):=\left(\pi_{2 i-j}^{X}\right)_{*} A^{i}(X),
$$

one obtains a bigraded ring structure on the Chow ring: that is, the intersection product sends $A_{(j)}^{i}(X) \otimes A_{\left(j^{\prime}\right)}^{i^{\prime}}(X)$ to $A_{\left(j+j^{\prime}\right)}^{i+i^{\prime}}(X)$.

It is expected (but not proven!) that for any $X$ with a weak MCK decomposition, one has

$$
A_{(j)}^{i}(X) \stackrel{? ?}{=} 0 \text { for } j<0, \quad A_{(0)}^{i}(X) \cap A_{\text {hom }}^{i}(X) \stackrel{? ?}{=} 0 ;
$$

this is related to Murre's conjectures $B$ and $D$, that have been formulated for any CK decomposition [30].

The property of having an MCK decomposition is severely restrictive, and is closely related to Beauville's “(weak) splitting property” [5]. For more ample discussion, and examples of varieties with an MCK decomposition, we refer to [38, Section 8], as well as [41], [39], [13].

Lemma 2.7. Let $X, X^{\prime}$ be birational hyperkähler varieties. Then $X$ has an MCK decomposition if and only if $X^{\prime}$ has one.

Proof. This is noted in [41, Introduction]; the idea is that Rieß's result [34] implies that $X$ and $X^{\prime}$ have isomorphic Chow motives and the isomorphism is compatible with the multiplicative structure.

\subsection{Relative MCK for $S^{[m]}$ and for $S^{m}$.}

Theorem 2.8 (Vial [41]). Let $S$ be a projective $K 3$ surface, and let $X=S^{[m]}$ be the Hilbert scheme of length $m$ subschemes of $S$. Then $X$ has a self-dual MCK decomposition $\left\{\Pi_{i}^{X}\right\}$. In particular, $A^{*}(X)=A_{(*)}^{*}(X)$ is a bigraded ring, where

$$
A^{i}(X)=\bigoplus_{j=2 i-2 n}^{i} A_{(j)}^{i}(X),
$$

and $A_{(j)}^{i}(X)=0$ for $j$ odd. In case $m=2$, the bigrading $A_{(*)}^{*}(X)$ coincides with the one given by the Fourier decomposition of theorem 2.1. 
Proof. This is [41, Theorems 1 and 2]. The last statement is [38, Theorem 15.8], plus the fact that for $m=2$ the MCK decomposition of [41] coincides with the one of [38].

Notation 2.9. Let $\mathcal{S} \rightarrow B$ be a family (i.e., a smooth projective morphism). For $r \in \mathbb{N}$, we write $\mathcal{S}^{r / B}$ for the relative $r$-fold fibre product

$$
\mathcal{S}^{r / B}:=\mathcal{S} \times{ }_{B} \mathcal{S} \times{ }_{B} \cdots \times{ }_{B} \mathcal{S}
$$

(r copies of $\mathcal{S})$.

Proposition 2.10. Let $\mathcal{S} \rightarrow B$ be a family of $K 3$ surfaces. There exist relative correspondences

$$
\Pi_{j}^{\mathcal{S}^{m / B}} \in A^{2 m}\left(\mathcal{S}^{m / B} \times \mathcal{S}^{m / B}\right) \quad(j=0,2,4, \ldots, 4 m),
$$

such that for each $b \in B$, the restriction

$$
\Pi_{j}^{\left(S_{b}\right)^{m}}:=\left.\Pi_{j}^{\mathcal{S}^{m / B}}\right|_{\left(S_{b}\right)^{2 m}} \in A^{4}\left(\left(S_{b}\right)^{m} \times\left(S_{b}\right)^{m}\right)
$$

defines a self-dual MCK decomposition for $\left(S_{b}\right)^{m}$.

Proof. On any $K 3$ surface $S_{b}$, there is the distinguished 0-cycle $\mathfrak{o}_{S_{b}}$ such that $c_{2}\left(S_{b}\right)=24 \mathfrak{o}_{S_{b}}$ [4]. Let $p_{i}: \mathcal{S}^{m / B} \rightarrow \mathcal{S}, i=1, \ldots, m$, denote the projections to the two factors. Let $T_{\mathcal{S} / B}$ denote the relative tangent bundle. The assignment

$$
\begin{aligned}
& \Pi_{0}^{\mathcal{S}}:=\left(p_{1}\right)^{*}\left(\frac{1}{24} c_{2}\left(T_{\mathcal{S} / B}\right)\right) \quad A^{2}\left(\mathcal{S} \times{ }_{B} \mathcal{S}\right), \\
& \Pi_{4}^{\mathcal{S}}:=\left(p_{2}\right)^{*}\left(\frac{1}{24} c_{2}\left(T_{\mathcal{S} / B}\right)\right) \quad A^{2}\left(\mathcal{S} \times{ }_{B} \mathcal{S}\right), \\
& \Pi_{2}^{\mathcal{S}}:=\Delta_{\mathcal{S}}-\Pi_{0}^{\mathcal{S}}-\Pi_{4}^{\mathcal{S}}
\end{aligned}
$$

defines (by restriction) an MCK decomposition for each fibre, i.e.

$$
\Pi_{j}^{S_{b}}:=\left.\Pi_{j}^{\mathcal{S}}\right|_{S_{b} \times S_{b}} \quad \in A^{2}\left(S_{b} \times S_{b}\right) \quad(j=0,2,4)
$$

is an MCK decomposition for any $b \in B$ [38, Example 8.17].

Next, we consider the $m$-fold relative fibre product $\mathcal{S}^{m / B}$. Let

$$
p_{i, j}: \mathcal{S}^{2 m / B} \rightarrow \mathcal{S}^{2 / B} \quad(1 \leq i<j \leq 2 m)
$$

denote projection to the $i$-th and $j$-th factor. We define

$$
\begin{array}{r}
\Pi_{j}^{\mathcal{S}^{m / B}}:=\sum_{k_{1}+k_{2}+\cdots+k_{m}=j}\left(p_{1, m+1}\right)^{*}\left(\Pi_{k_{1}}^{\mathcal{S}}\right) \cdot\left(p_{2, m+2}\right)^{*}\left(\Pi_{k_{2}}^{\mathcal{S}}\right) \cdot \ldots \cdot\left(p_{m, 2 m}\right)^{*}\left(\Pi_{k_{m}}^{\mathcal{S}}\right) \\
\in A^{2 m}\left(\mathcal{S}^{2 m / B}\right), \quad(j=0,2,4, \ldots, 4 m) .
\end{array}
$$

By construction, the restriction to each fibre induces an MCK decomposition (the "product MCK decomposition")

$$
\begin{aligned}
& \Pi_{j}^{\left(S_{b}\right)^{m}}:=\left.\Pi_{j}^{\mathcal{S}^{m / B}}\right|_{\left(S_{b}\right)^{2 m}}=\sum_{k_{1}+k_{2}+\cdots+k_{m}=j} \Pi_{k_{1}}^{S_{b}} \times \Pi_{k_{2}}^{S_{b}} \times \cdots \times \Pi_{k_{m}}^{S_{b}} \in A^{2 m}\left(\left(S_{b}\right)^{2 m}\right), \\
& (j=0,2,4, \ldots, 4 m) \text {. }
\end{aligned}
$$


Remark 2.11. Let $\mathcal{S} \rightarrow B$ be a family of $K 3$ surfaces. Let $\mathcal{X} \rightarrow B$ denote the relative Hilbert scheme (i.e., the blow-up of $\mathcal{S} \times{ }_{B} \mathcal{S}$ along the relative diagonal, quotiented by the natural action of the symmetric group on 2 elements $\mathfrak{S}_{2}$ ). Since the above construction of the $\Pi_{j}^{\mathcal{S}^{2 / B}}$ is $\mathfrak{S}_{2}-$ invariant, it induces relative correspondences

$$
\Pi_{j}^{\mathcal{X}} \in A^{4}\left(\mathcal{X} \times{ }_{B} \mathcal{X}\right) \quad(j=0,2,4,6,8),
$$

with the property that the restrictions

$$
\Pi_{j}^{X_{b}}:=\left.\Pi_{j}^{\mathcal{X}}\right|_{X_{b} \times X_{b}} \in A^{4}\left(X_{b} \times X_{b}\right)
$$

form an MCK decomposition for $X_{b}$, for all $b \in B$ (cf. [25, Proposition 2.16] for a detailed proof).

Proposition 2.12. Let $\mathcal{S} \rightarrow B$ be a family of $K 3$ surfaces. There exist relative correspondences

$$
\Theta_{1}^{\prime}, \ldots, \Theta_{m}^{\prime} \in A^{2 m}\left(\mathcal{S}^{m / B} \times_{B} \mathcal{S}\right), \quad \Xi_{1}^{\prime}, \ldots, \Xi_{m}^{\prime} \in A^{2}\left(\mathcal{S} \times_{B} \mathcal{S}^{m / B}\right)
$$

such that for each $b \in B$, the composition

$$
\begin{aligned}
& A_{(2)}^{2 m}\left(\left(S_{b}\right)^{m}\right) \stackrel{\left(\left(\left.\Theta_{1}^{\prime}\right|_{\left.\left.\left(S_{b}\right)^{m+1}\right)_{*}, \ldots,\left(\left.\Theta_{m}^{\prime}\right|_{\left(S_{b}\right)^{m+1}}\right)_{*}\right)} ^{\longrightarrow}\right.\right.}{\longrightarrow} A^{2}\left(S_{b}\right) \oplus \cdots \oplus A^{2}\left(S_{b}\right) \\
& \stackrel{\left(\left.\left(\Xi_{1}^{\prime}+\ldots+\Xi_{m}^{\prime}\right)\right|_{\left.\left(S_{b}\right)^{m+1}\right)_{*}} ^{\longrightarrow}\right.}{\longrightarrow} A^{2 m}\left(\left(S_{b}\right)^{m}\right)
\end{aligned}
$$

is the identity.

Proof. As before, let

$$
p_{i, j}: \quad \mathcal{S}^{2 m / B} \rightarrow \mathcal{S}^{2 / B} \quad(1 \leq i<j \leq 2 m)
$$

denote projection to the $i$-th and $j$-th factor, and let

$$
p_{i}: \mathcal{S}^{m / B} \rightarrow \mathcal{S} \quad(1 \leq i \leq m)
$$

denote projection to the $i$-th factor.

We now claim that for each $b \in B$, there is equality

(1)

$$
\begin{aligned}
&\left.\left(\Pi_{4 m-2}^{\mathcal{S}^{m / B}}\right)\right|_{\left(S_{b}\right)^{2 m}}=\frac{1}{24^{2 m-2}}\left({ }^{t} \Gamma_{p_{1}} \circ \Pi_{2}^{\mathcal{S}} \circ \Gamma_{p_{1}} \circ\left(\left(p_{1, m+1}\right)^{*}\left(\Delta_{\mathcal{S}}\right) \cdot \prod_{\substack{2 \leq j \leq 2 m \\
j \neq m+1}}\left(p_{j}\right)^{*} c_{2}\left(T_{\mathcal{S} / B}\right)\right)\right. \\
&\left.+\ldots+{ }^{t} \Gamma_{p_{m}} \circ \Pi_{2}^{\mathcal{S}} \circ \Gamma_{p_{m}} \circ\left(\left(p_{m, 2 m}\right)^{*}\left(\Delta_{\mathcal{S}}\right) \cdot \prod_{\substack{1 \leq j \leq 2 m-1 \\
j \neq m}}\left(p_{j}\right)^{*} c_{2}\left(T_{\mathcal{S} / B}\right)\right)\right)\left.\right|_{\left(S_{b}\right)^{2 m}} \quad \text { in } A^{2 m}\left(\left(S_{b}\right)^{m} \times\left(S_{b}\right)^{m}\right) .
\end{aligned}
$$

Indeed, using Lieberman's lemma [14, 16.1.1], we find that

$$
\begin{gathered}
\left.\left({ }^{t} \Gamma_{p_{1}} \circ \Pi_{2}^{\mathcal{S}} \circ \Gamma_{p_{1}}\right)\right|_{\left(S_{b}\right)^{2 m}}=\left.\left(\left({ }^{t} \Gamma_{p_{1, m+1}}\right)_{*}\left(\Pi_{2}^{\mathcal{S}}\right)\right)\right|_{\left(S_{b}\right)^{2 m}}=\left.\left(\left(p_{1, m+1}\right)^{*}\left(\Pi_{2}^{\mathcal{S}}\right)\right)\right|_{\left(S_{b}\right)^{2 m}}, \\
\vdots \\
\left.\left({ }^{t} \Gamma_{p_{m}} \circ \prod_{2}^{\mathcal{S}} \circ \Gamma_{p_{m}}\right)\right|_{\left(S_{b}\right)^{2 m}}=\left.\left(\left({ }^{t} \Gamma_{p_{m, 2 m}}\right)_{*}\left(\Pi_{2}^{\mathcal{S}}\right)\right)\right|_{\left(S_{b}\right)^{2 m}}=\left.\left(\left(p_{m, 2 m}\right) *\left(\Pi_{2}^{\mathcal{S}}\right)\right)\right|_{\left(S_{b}\right)^{2 m}} .
\end{gathered}
$$


Let us now (by way of example) consider the first summand of the right-hand side of (1). For brevity, let

$$
P: \quad\left(S_{b}\right)^{3 m} \rightarrow\left(S_{b}\right)^{2 m}
$$

denote the projection on the first $m$ and last $m$ factors. Writing out the definition of composition of correspondences, we find that

$$
\begin{aligned}
& \left.\frac{1}{24^{2 m-2}}\left({ }^{t} \Gamma_{p_{1}} \circ \Pi_{2}^{\mathcal{S}} \circ \Gamma_{p_{1}} \circ\left(\left(p_{1, m+1}\right)^{*}\left(\Delta_{\mathcal{S}}\right) \cdot \prod_{\substack{2 \leq j \leq 2 m \\
j \neq m+1}}\left(p_{j}\right)^{*} c_{2}\left(T_{\mathcal{S} / B}\right)\right)\right)\right|_{\left(S_{b}\right)^{2 m}}= \\
& \frac{1}{24^{2 m-2}}\left(\left(\left(p_{1, m+1}\right)^{*}\left(\Pi_{2}^{S_{b}}\right)\right) \circ\left(\left(p_{1, m+1}\right)^{*}\left(\Delta_{S_{b}}\right) \cdot \prod_{\substack{2 \leq j \leq 2 m \\
j \neq m+1}}\left(p_{j}\right)^{*} c_{2}\left(T_{S_{b}}\right)\right)\right)= \\
& P_{*}\left(\left(\left(\Delta_{S_{b}}\right)_{(1, m+1)} \times \mathfrak{o}_{S_{b}} \times \cdots \times \mathfrak{o}_{S_{b}} \times S_{b} \times \cdots \times S_{b}\right) \cdot\right. \\
& \left.\quad\left(S_{b} \times \cdots \times S_{b} \times\left(\Pi_{2}^{S_{b}}\right)_{(m+1,2 m+1)} \times S_{b} \times \cdots \times S_{b}\right)\right)= \\
& P_{*}\left(\left(\left(\Delta_{S_{b}} \times S_{b}\right) \cdot\left(S_{b} \times \Pi_{2}^{S_{b}}\right)\right)_{(1, m+1,2 m+1)} \times \mathfrak{o}_{S_{b}} \times \cdots \times \mathfrak{o}_{S_{b}} \times S_{b} \times \cdots \times S_{b}\right)= \\
& \prod_{2}^{S_{b}} \times \Pi_{4}^{S_{b}} \times \cdots \times \Pi_{4}^{S_{b}} \quad \text { in } A^{2 m}\left(\left(S_{b}\right)^{m} \times\left(S_{b}\right)^{m}\right) .
\end{aligned}
$$

(Here, we use the notation $(C)_{(i, j)}$ to indicate that the cycle $C$ lies in the $i$ th and $j$ th factor, and likewise for $(D)_{(i, j, k)}$.)

Doing the same for the other summands in (1), one convinces oneself that both sides of (1) are equal to the fibrewise product Chow-Künneth component

$$
\Pi_{4 m-2}^{\left(S_{b}\right)^{m}}=\Pi_{2}^{S_{b}} \times \Pi_{4}^{S_{b}} \times \cdots \times \Pi_{4}^{S_{b}}+\cdots+\Pi_{4}^{S_{b}} \times \cdots \times \Pi_{4}^{S_{b}} \times \Pi_{2}^{S_{b}} \in A^{2 m}\left(\left(S_{b}\right)^{m} \times\left(S_{b}\right)^{m}\right),
$$

thus proving the claim.

Let us now define

$$
\begin{aligned}
& \Theta_{i}^{\prime}:=\frac{1}{24^{2 m-2}} \Gamma_{p_{i}} \circ\left(\left(p_{i, m+i}\right)^{*}\left(\Delta_{\mathcal{S}}\right) \cdot \prod_{\substack{j \in[1,2 m] \\
j \notin\{i, m+i\}}}\left(p_{j}\right)^{*} c_{2}\left(T_{\mathcal{S} / B}\right)\right) \in A^{2 m}\left(\left(\mathcal{S}^{m / B}\right) \times{ }_{B} \mathcal{S}\right), \\
& \Xi_{i}^{\prime}:={ }^{t} \Gamma_{p_{i}} \circ \Pi_{2}^{\mathcal{S}} \in A^{2}\left(\mathcal{S} \times{ }_{B}\left(\mathcal{S}^{m / B}\right)\right),
\end{aligned}
$$

where $1 \leq i \leq m$. It follows from equation (1) that there is equality

$$
\begin{aligned}
\left(\left.\left(\Xi_{1}^{\prime} \circ \Theta_{1}^{\prime}+\cdots+\Xi_{m}^{\prime} \circ \Theta_{m}^{\prime}\right)\right|_{\left(S_{b}\right)^{2 m}}\right) * & =\left(\Pi_{4 m-2}^{\left(S_{b}\right)^{m}}\right)_{*}: \\
A_{(j)}^{i}\left(\left(S_{b}\right)^{m}\right) & \rightarrow A_{(j)}^{i}\left(\left(S_{b}\right)^{m}\right) \quad \forall b \in B \quad \forall(i, j) .
\end{aligned}
$$

Taking $(i, j)=(2 m, 2)$, this proves the proposition.

The following is a version of proposition 2.12 for the group $A_{(2)}^{2}\left(\left(S_{b}\right)^{m}\right)$ :

Proposition 2.13. Let $\mathcal{S} \rightarrow B$ be a family of $K 3$ surfaces. There exist relative correspondences

$$
\Theta_{1}, \ldots, \Theta_{m} \in A^{2 m}\left(\mathcal{S} \times_{B}\left(\mathcal{S}^{m / B}\right)\right), \quad \Xi_{1}, \ldots, \Xi_{m} \in A^{2}\left(\left(\mathcal{S}^{m / B}\right) \times_{B} \mathcal{S}\right)
$$


such that for each $b \in B$, the composition

$$
A_{(2)}^{2}\left(\left(S_{b}\right)^{m}\right) \stackrel{\left(\left(\left.\Xi_{1}\right|_{\left.\left(S_{b}\right)^{m+1}\right)_{*}, \ldots,\left(\left.\Xi_{m}\right|_{\left.\left.\left(S_{b}\right)^{m+1}\right)_{*}\right)}\right.} A^{2}\left(S_{b}\right) \oplus \cdots \oplus A^{2}\left(S_{b}\right)\right.\right.}{\stackrel{\left(\left.\left(\Theta_{1}+\ldots+\Theta_{m}\right)\right|_{\left.\left(S_{b}\right)^{m+1}\right)_{*}}\right.}{\longrightarrow} A^{2}\left(\left(S_{b}\right)^{m}\right)}
$$

is the identity.

Proof. One may take

$$
\begin{aligned}
& \Theta_{i}:={ }^{t} \Theta_{i}^{\prime} \quad \in A^{2 m}\left(\mathcal{S} \times{ }_{B}\left(\mathcal{S}^{m / B}\right)\right) \\
& \Xi_{i}:={ }^{t} \Xi_{i}^{\prime} \quad A^{2}\left(\left(\mathcal{S}^{m / B}\right) \times{ }_{B} \mathcal{S}\right) \quad(i=1, \ldots, m) .
\end{aligned}
$$

By construction, the product MCK decomposition $\left\{\Pi_{i}^{\left(S_{b}\right)^{m}}\right\}$ satisfies

$$
\Pi_{2}^{\left(S_{b}\right)^{m}}={ }^{t}\left(\Pi_{4 m-2}^{\left(S_{b}\right)^{m}}\right) \text { in } A^{2 m}\left(\left(S_{b}\right)^{m} \times\left(S_{b}\right)^{m}\right) .
$$

Hence, the transpose of equation (2) gives the equality

$$
\begin{aligned}
\left(\Pi_{2}^{\left(S_{b}\right)^{m}}\right)_{*}=\left({ }^{t}\left(\Pi_{4 m-2}^{\left(S_{b}\right)^{m}}\right)\right)_{*}=\left({ }^{t} \Theta_{1}^{\prime} \circ{ }^{t} \Xi_{1}^{\prime}+\ldots+{ }^{t} \Theta_{m}^{\prime} \circ{ }^{t} \Xi_{m}^{\prime}\right)_{*}: & \\
A_{(j)}^{i}\left(\left(S_{b}\right)^{m}\right) & \rightarrow A_{(j)}^{i}\left(\left(S_{b}\right)^{m}\right) \quad \forall b \in B \quad \forall(i, j) .
\end{aligned}
$$

Taking $(i, j)=(2,2)$, this proves the proposition.

\subsection{Relative CK for Fano varieties.}

Theorem 2.14 (Shen-Vial [38]). Let $Y \subset \mathbb{P}^{5}(\mathbb{C})$ be a smooth cubic, and let $X=F(Y)$ be the Fano variety of lines in $X$. The Fourier decomposition is induced by a $C K$ decomposition $\left\{\Pi_{i}^{X}\right\}$, i.e. there is equality

$$
A_{(j)}^{i}(X)=\left(\Pi_{2 i-j}^{X}\right)_{*} A^{i}(X) \quad \forall i, j .
$$

In particular, for very general $Y$, the $\left\{\Pi_{i}^{X}\right\}$ are a weak MCK decomposition.

Proof. This is [38, Theorem 3.3]. The last statement follows from theorem 2.2(ii).

Notation 2.15. Let

$$
\mathcal{Y} \rightarrow B
$$

denote the universal family of smooth cubic fourfolds $Y_{b}$. Here $B$ is a Zariski open in the parameter space $\mathbb{P} H^{0}\left(\mathbb{P}^{5}, \mathcal{O}_{\mathbb{P}^{5}}(3)\right)$. Let

$$
\mathcal{X}:=\left\{(\ell, b) \mid \ell \subset Z_{b}\right\} \quad \subset G(1,5) \times B
$$

be the corresponding family of Fano varieties of lines. (Here $G(1,5)$ denotes the Grassmannian of lines in $\mathbb{P}^{5}$.) A fibre $X_{b}$ of $\mathcal{X} \rightarrow B$ is a Fano variety of lines on a cubic $Y_{b}$.

Proposition 2.16. Let $\mathcal{X} \rightarrow B$ be as in notation 2.15. There exist relative correspondences

$$
\Pi_{i}^{\mathcal{X}} \in A^{4}\left(\mathcal{X} \times_{B} \mathcal{X}\right) \quad(i=2,6)
$$

with the property that for each $b \in B$, one has

$$
\begin{array}{ll}
\left(\left.\left(\Pi_{2}^{\mathcal{X}}\right)\right|_{X_{b} \times X_{b}}\right)_{*}=\left(\Pi_{2}^{X_{b}}\right)_{*}: & A^{2}\left(X_{b}\right) \rightarrow A^{2}\left(X_{b}\right), \\
\left(\left.\left(\Pi_{6}^{\mathcal{X}}\right)\right|_{X_{b} \times X_{b}}\right)_{*}=\left(\Pi_{6}^{X_{b}}\right)_{*}: & A^{4}\left(X_{b}\right) \rightarrow A^{4}\left(X_{b}\right) .
\end{array}
$$


Here $\Pi_{i}^{X_{b}}$ is the Chow-Künneth decomposition of theorem 2.2(i).

Proof. The main point is that the Shen-Vial cycle $L \in A^{2}\left(X_{b} \times X_{b}\right)$ furnishing the Fourier decomposition [38] exists relatively: this is because by definition

$$
L:=\frac{1}{3}\left(g_{1}^{2}+\frac{3}{2} g_{1} g_{2}+g_{2}^{2}-c_{1}-c_{2}\right)-I \quad \in A^{2}\left(X_{b} \times X_{b}\right)
$$

[38, Equation (107)]. Here, $g:=-c_{1}\left(\mathcal{E}_{2}\right) \in A^{1}\left(X_{b}\right)$ and $c:=c_{2}\left(\mathcal{E}_{2}\right) \in A^{2}\left(X_{b}\right)$ (and $\mathcal{E}_{2}$ is the restriction of the rank 2 tautological bundle on the Grassmannian), and $g_{i}:=\left(p_{i}\right)^{*}(g)$, $c_{i}:=\left(p_{i}\right)^{*}(c)$ (where $p_{i}: X_{b} \times X_{b} \rightarrow X_{b}$ is projection on the $i$ th factor), and $I \subset X_{b} \times X_{b}$ is the incidence correspondence. Since $g_{i}, c_{i}$ and $I$ obviously exist relatively, the same goes for $L$, i.e. there exists a relative correspondence

$$
\mathcal{L} \in A^{2}\left(\mathcal{X} \times_{B} \mathcal{X}\right)
$$

with the property that for any $b \in B$ the restriction

$$
\left.\mathcal{L}\right|_{X_{b} \times X_{b}} \in A^{2}\left(X_{b} \times X_{b}\right)
$$

is the Shen-Vial class $L$ of [38]. This implies that the class $\ell \in A^{2}\left(X_{b}\right)$ of [38] (mentioned in theorem 2.17(ii) below) also exists relatively: it is defined as

$$
\ell:=\left(i_{\Delta}\right)^{*}(\mathcal{L}) \quad \in A^{2}(\mathcal{X})
$$

where $i_{\Delta}: \mathcal{X} \rightarrow \mathcal{X} \times_{B} \mathcal{X}$ denotes the embedding along the relative diagonal. (NB: this makes sense because $i_{\Delta}$ is a regular embedding.) Next, the classes $\ell_{i}:=\left(p_{i}\right)^{*}(\ell) \in A^{2}\left(X_{b} \times X_{b}\right)$ of [38] also exist relatively.

Armed with these facts, let us inspect the construction of the $\left\{\Pi_{i}^{X_{b}}\right\}$ in [38, Theorem 3.3].

As a first approach towards the construction of $\Pi_{2}^{X_{b}}$ and $\Pi_{6}^{X_{b}}$, Shen-Vial define

$$
p_{b}:=\frac{1}{25} L \cdot \ell_{2} \quad \in A^{4}\left(X_{b} \times X_{b}\right) .
$$

Again, by the above remarks the cycle $p_{b}$ exists relatively (i.e. there is $\mathfrak{p} \in A^{4}\left(\mathcal{X} \times{ }_{B} \mathcal{X}\right)$ which restricts to $p_{b}$ on each fibre). We define $\Pi_{6}^{\mathcal{X}}:=\mathfrak{p} \in A^{4}\left(\mathcal{X} \times_{B} \mathcal{X}\right)$. This does the job, for it is shown in [38, Proof of Theorem 3.3] that $\left(p_{b}\right)_{*}$ acts as the identity on $A_{(2)}^{4}\left(X_{b}\right)$ and acts as 0 on $A_{(0)}^{4}\left(X_{b}\right) \oplus A_{(4)}^{4}\left(X_{b}\right)$.

We define $\Pi_{2}^{\mathcal{X}}$ as the transpose $\Pi_{2}^{\mathcal{X}}:={ }^{t} \Pi_{6}^{\mathcal{X}}$. This does the job, for it is shown in loc. cit. that $\left(p_{b}\right)^{*}$ acts as the identity on $A_{(2)}^{2}\left(X_{b}\right)$ and acts as 0 on $A_{(0)}^{2}\left(X_{b}\right)$.

\subsection{A result in the Chow ring of $S^{[2]}$.}

Theorem 2.17 (Shen-Vial [38]). Let $X=S^{[2]}$ where $S$ is a K3 surface. There exists a class $\ell \in A_{(0)}^{2}(X)$ inducing an isomorphism

$$
\cdot \ell: \quad A_{(2)}^{2}(X) \stackrel{\cong}{\rightarrow} A_{(2)}^{4}(X) .
$$

The inverse isomorphism is given by

$$
\frac{1}{25} L_{*}: \quad A_{(2)}^{4}(X) \stackrel{\cong}{\rightarrow} A_{(2)}^{2}(X),
$$


where $L \in A^{2}(X \times X)$ is the Shen-Vial class lifting the Beauville-Bogomolov form.

Proof. This is [38, Theorems 2.2 and 2.4] (combined with the fact that $X$ satisfies the hypotheses of loc. cit., which is [38, Part 2].

For later use, we remark that the Shen-Vial class $L$ exists relatively:

Lemma 2.18. Let $\mathcal{S} \rightarrow B$ be a family of $K 3$ surfaces, and let $\mathcal{X} \rightarrow B$ be the family of Hilbert squares. There exists a class $\mathcal{L} \in A^{2}\left(\mathcal{X} \times_{B} \mathcal{X}\right)$ such that for any $b \in B$, the restriction

$$
L_{b}:=\left.\mathcal{L}\right|_{X_{b} \times X_{b}} \quad \in A^{2}\left(X_{b} \times X_{b}\right)
$$

is the Shen-Vial class of theorem 2.17.

Proof. For a given $K 3$ surface $S=S_{b}$, let

$$
\begin{array}{lll}
Z & \stackrel{q}{\rightarrow} S \\
\downarrow p & & \\
X & &
\end{array}
$$

denote the universal family of length 2 subschemes of $S$. Let

$$
I:={ }^{t} Z \circ Z \in A^{2}(X \times X)
$$

denote the incidence correspondence. Let $\delta \in A^{1}(X)$ be the class supported on the exceptional divisor as in [38, Section 10], and let $\delta_{i}:=\left(p_{i}\right)^{*}(\delta) \in A^{1}(X \times X)$, where $p_{i}: X \times X \rightarrow X$ is projection on the $i$ th factor. Let $\mathfrak{o}_{S} \in A^{2}(S)$ denote the distinguished point [4], and let $S_{\mathfrak{o}}:=$ $p_{*} q^{*}\left(\mathfrak{o}_{S}\right) \in A^{2}(X)$.

By definition, the Shen-Vial class $L$ is

$$
L=I-2\left(p_{1}\right)^{*}\left(S_{\mathfrak{o}}\right)-2\left(p_{2}\right)^{*}\left(S_{\mathfrak{o}}\right)-\frac{1}{2} \delta_{1} \delta_{2} \in A^{2}(X \times X)
$$

[38, Equation (92)].

The distinguished point $\mathfrak{o}_{S}$ equals $\frac{1}{24} c_{2}\left(T_{S}\right)$ [4], hence it can be defined relatively. The universal family $Z$ can also be defined relatively, hence the same holds for the incidence correspondence $I$. This implies that the class $L$ can be defined relatively, i.e. there exists a class $\mathcal{L}$ as indicated.

\subsection{A result in the Chow ring of the Fano variety.}

Theorem 2.19 (Shen-Vial [38]). Let $Z \subset \mathbb{P}^{5}(\mathbb{C})$ be a smooth cubic fourfold, and let $X$ be the Fano variety of lines in $Z$. There is a distinguished class $l \in A_{(0)}^{2}(X)$ such that intersection induces an isomorphism

$$
\cdot l: \quad A_{(2)}^{2}(X) \stackrel{\cong}{\rightarrow} A_{(2)}^{4}(X) .
$$

The inverse isomorphism is given by

$$
\frac{1}{25} L_{*}: \quad A_{(2)}^{4}(X) \stackrel{\cong}{\rightarrow} A_{(2)}^{2}(X),
$$

where $L \in A^{2}(X \times X)$ is the Shen-Vial class lifting the Beauville-Bogomolov form. 
Proof. This follows from [38, Theorems 2.2 and 2.4] (combined with the fact that $X$ satisfies the hypotheses of loc. cit., which is [38, Part 3].

The following is a reformulation of theorem 2.19:

Proposition 2.20. Let $Y \subset \mathbb{P}^{5}(\mathbb{C})$ be a smooth cubic fourfold, and let $X$ be the Fano variety of lines in $Y$. Let $I \in A^{2}(X \times X)$ be the incidence correspondence, and let $g=-c_{1}\left(\mathcal{E}_{2}\right) \in A^{1}(X)$ be the Plücker polarization. Then

$$
\cdot g^{2}: \quad A_{(2)}^{2}(X) \rightarrow A_{(2)}^{4}(X)
$$

is an isomorphism. The inverse isomorphism is given by

$$
-\frac{1}{6} I_{*}: \quad A_{(2)}^{4}(X) \rightarrow A_{(2)}^{2}(X) .
$$

Proof. This is implicit in the arguments of [38]. For any $a \in A_{h o m}^{4}(X)$, there is equality

$$
\ell \cdot L_{*}(a)=-\frac{25}{6} g^{2} \cdot I_{*}(a) \text { in } A^{4}(X) .
$$

(This follows from [38, Equations (107) and (108)], cf. the proof of [38, Proposition 19.4].) But for $a \in A_{(2)}^{4}(X)$, we know (theorem 2.17(ii)) that $\ell \cdot L_{*}(a)=25 a$, and so for any $a \in A_{(2)}^{4}(X)$ we get an equality

$$
a=-\frac{1}{6} g^{2} \cdot I_{*}(a) \text { in } A^{4}(X) .
$$

Applying $I_{*}$ to this equality, we obtain an equality (for any $a \in A_{(2)}^{4}(X)$ )

$$
I_{*}(a)=-\frac{1}{6} I_{*}\left(g^{2} \cdot I_{*}(a)\right) \text { in } A^{2}(X) .
$$

But we know that

$$
A_{(2)}^{2}(X)=I_{*} A_{\text {hom }}^{4}(X)=I_{*} A_{(2)}^{4}(X) .
$$

(Here, the first equality is [38, Proof of Proposition 21.10], and the second equality follows from the fact that $I_{*} A_{(4)}^{4}(X)=0$ [38, Theorem 20.5].) Equation (3) thus becomes the statement that for any $b \in A_{(2)}^{2}(X)$ there is equality

$$
b=-\frac{1}{6} I_{*}\left(g^{2} \cdot b\right) \quad \text { in } A^{2}(X) .
$$

This proves the proposition.

Corollary 2.21. Let $Y \subset \mathbb{P}^{5}(\mathbb{C})$ be a smooth cubic fourfold, and let $X$ be the Fano variety of lines in $Y$. There exist correspondences $P \in A^{3}(X \times Y), Q \in A^{5}(Y \times X)$ such that

$$
\begin{aligned}
\left({ }^{t} P \circ{ }^{t} Q\right)_{*}=\mathrm{id}: & A_{(2)}^{2}(X) \rightarrow A_{(2)}^{2}(X), \\
(Q \circ P)_{*}=\mathrm{id}: & A_{(2)}^{4}(X) \rightarrow A_{(2)}^{4}(X) .
\end{aligned}
$$


Moreover, let $\mathcal{Y} \rightarrow B$ and $\mathcal{X} \rightarrow B$ denote the universal families as in notation 2.29. Then there exist relative correspondences $\mathcal{P} \in A^{3}\left(\mathcal{X} \times_{B} \mathcal{Y}\right)$ and $\mathcal{Q} \in A^{5}\left(\mathcal{Y} \times_{B} \mathcal{X}\right)$ with the above property on each fibre: i.e., for each $b \in B$ we have

$$
\begin{aligned}
& \left(\left.\left({ }^{t} \mathcal{P} \circ{ }^{t} \mathcal{Q}\right)\right|_{X_{b} \times X_{b}}\right)_{*}=\left(\Pi_{2}^{X_{b}}\right)_{*}: \quad A^{2}\left(X_{b}\right) \rightarrow A^{2}\left(X_{b}\right), \\
& \left(\left.(\mathcal{Q} \circ \mathcal{P})\right|_{X_{b} \times X_{b}}\right)_{*}=\left(\Pi_{6}^{X_{b}}\right)_{*}: \quad A^{4}\left(X_{b}\right) \rightarrow A^{4}\left(X_{b}\right) \text {. }
\end{aligned}
$$

Proof. The correspondence $P \in A^{3}(X \times Y)$ is defined as the universal family of lines on $Y$. Letting $I \subset X \times X$ denote the incidence correspondence, we have

$$
I={ }^{t} P \circ P \text { in } A^{2}(X \times X)
$$

[38, Lemma 17.2].

Proposition 2.20 states that the composition

$$
A_{(2)}^{2}(X) \stackrel{\cdot g^{2}}{\longrightarrow} A_{(2)}^{4}(X) \stackrel{-\frac{1}{6}\left({ }^{t} P \circ P\right)_{*}}{\longrightarrow} A_{(2)}^{2}(X)
$$

is the identity, in other words

$$
\left.\left(-\frac{1}{6} t\right) P \circ P \circ \Gamma_{g^{2}}\right)_{*}=\mathrm{id}: \quad A_{(2)}^{2}(X) \rightarrow A_{(2)}^{2}(X) .
$$

(Here $\Gamma_{g^{2}} \in A^{6}(X \times X)$ can be defined as $\frac{1}{d}{ }^{t} \Gamma_{\tau} \circ \Gamma_{\tau}$, where $\tau: R \rightarrow X$ denotes the inclusion of a smooth complete intersection of class $\left.d g^{2}\right)$. Defining

$$
{ }^{t} Q:=-\frac{1}{6} P \circ \Gamma_{g^{2}} \in A^{5}(X \times Y),
$$

we obtain the first equality of corollary 2.21 .

For the second equality, it suffices to take the transpose of the first equality, since we know that the CK decomposition of theorem 2.14 is self-dual.

As to the "moreover" part of the corollary: obviously both $P$ and $\Gamma_{g^{2}}$ exist relatively, and so the same goes for $Q$.

\subsection{Spread.}

Proposition 2.22 (Voisin [43]). Let $M$ be a smooth projective variety of dimension $r+2$, and assume $M$ has trivial Chow groups (i.e. $A_{\text {hom }}^{*}(M)=0$ ). Let $L_{1}, \ldots, L_{r}$ be very ample line bundles on $M$, and let

$$
\mathcal{Y} \rightarrow B
$$

be the universal family of smooth complete intersections

$$
Y_{b}=M \cap D_{1} \cap \cdots \cap D_{r}, \quad D_{j} \in\left|L_{j}\right| .
$$

Let $R \in A^{2}\left(\mathcal{Y} \times_{B} \mathcal{Y}\right)$ be a relative correspondence such that

$$
\left.R\right|_{Y_{b} \times Y_{b}}=0 \in H^{4}\left(Y_{b} \times Y_{b}\right) \text { for very general } b \in B .
$$

Then there exists $\delta \in A^{n}(M \times M)$ such that

$$
\left.R\right|_{Y_{b} \times Y_{b}}=\left.\delta\right|_{Y_{b} \times Y_{b}} \in A^{2}\left(Y_{b} \times Y_{b}\right) \quad \forall b \in B .
$$


Proof. This follows from [43] (NB: as noted in loc. cit., the "Voisin standard conjecture" [43, Conjecture 1.6] is satisfied for $n=2$, so is not needed as extra assumption).

(Alternatively, one could give a quick proof of proposition 2.22 along the lines of [44, Proposition 1.6], at least under the extra assumption that the surfaces $Y_{b}$ have non-zero primitive cohomology, which is OK in all cases where we apply proposition 2.22 since we only consider $K 3$ surfaces.)

\subsection{Families of $K 3$ surfaces.}

Notation 2.23. Let $g \in\{2,3,4,5,6,8\}$. Let

$$
\mathbb{P}_{g}:= \begin{cases}\mathbb{P}\left(1^{3}, 3\right) & \text { if } g=2, \\ \mathbb{P}^{g}(\mathbb{C}) & \text { if } g=3,4,5, \\ G(1,4) & \text { if } g=6 \\ G(1,5) & \text { if } g=8\end{cases}
$$

(here $\mathbb{P}\left(1^{3}, 3\right)$ denotes a weighted projective space, and $G(1, m)$ is the Grassmannian of lines in $\mathbb{P}^{m}(\mathbb{C})$ ). Consider the vector bundle $U_{g}$ on $\mathbb{P}_{g}$ defined as

$$
U_{g}:= \begin{cases}\mathcal{O}(6) & \text { if } g=2, \\ \mathcal{O}(4) & \text { if } g=3, \\ \mathcal{O}(3) \oplus \mathcal{O}(2) & \text { if } g=4, \\ \mathcal{O}(2)^{\oplus 3} & \text { if } g=5, \\ \mathcal{O}(2) \oplus \mathcal{O}(1)^{\oplus 3} & \text { if } g=6, \\ \mathcal{O}(1)^{\oplus 6} & \text { if } g=8\end{cases}
$$

(here $\mathcal{O}(i)$ on a Grassmannian refers to the Plücker embedding).

Let $B_{g} \subset \mathbb{P} H^{0}\left(\mathbb{P}_{g}, U_{g}\right)$ denote the Zariski open parametrizing smooth sections, and let

$$
\mathcal{S}_{g}:=\{(x, s) \mid s(x)=0\} \quad \subset \mathbb{P}_{g} \times B_{g}
$$

denote the universal family.

As shown by Mukai [29], a general $K 3$ surface of genus $g \in\{2,3,4,5,6,8\}$ is isomorphic to a fibre $S_{b}$ of the family $\mathcal{S}_{g} \rightarrow B_{g}$.

Notation 2.24. Let $\mathcal{S}_{g} \rightarrow B_{g}$ be as in notation 2.23. The family

$$
\mathcal{S}_{g}^{[2]} \rightarrow B_{g}
$$

is defined as follows: take $\mathcal{S} \times{ }_{B} \mathcal{S}$ and blow-up the relative diagonal, then take the quotient for the action of the symmetric group exchanging the two factors. The fibre of $\mathcal{S}_{g}^{[2]} \rightarrow B_{g}$ is the Hilbert square $\left(S_{b}\right)^{[2]}$ of the K3 surface $S_{b}$. 


\subsection{A Lagrangian fibration.}

Proposition 2.25 (Mukai [28]). Let $S$ be a general $K 3$ surface of genus 5 , and let $X=S^{[2]}$ be the Hilbert scheme. There exists a Lagrangian fibration

$$
\phi: \quad X \rightarrow \mathbb{P}^{2} .
$$

Proof. The surface $S$ can be defined as the intersection of three quadrics $Q_{1}, Q_{2}, Q_{3}$ in $\mathbb{P}^{5}(\mathbb{C})$. Let $N \cong\left(\mathbb{P}^{2}\right)^{\vee}$ be the net of quadrics spanned by $Q_{1}, Q_{2}, Q_{3}$. Any length 2 subscheme $\xi$ in $S$ determines a line $\ell_{\xi}$ in $\mathbb{P}^{5}$. Quadrics in $N$ containing the line $\ell_{\xi}$ form a pencil $P_{\xi} \cong \mathbb{P}^{1}$ inside $N$. Dually, this determines a point in $\mathbb{P}^{2}$, and so we obtain a morphism

$$
\begin{aligned}
\phi: X & \rightarrow \mathbb{P}^{2}, \\
\xi & \mapsto\left(P_{\xi}\right)^{\vee} .
\end{aligned}
$$

(This fibration $\phi$ is also described in [36, Section 2.1] and [9].)

Remark 2.26. Hassett-Tschinkel prove more generally that $X=S^{[2]}$ admits a Lagrangian fibration, for $S$ a generic $K 3$ surface of degree $2 m^{2}$ with $m \geq 2$ [18, Proposition 7.1]. Generalizations to certain higher-dimensional Hilbert schemes $S^{[r]}, r>2$ are given in [36] and [20].

2.10. A relation of motives. Galkin-Shinder [15] have constructed a relation between a cubic and its Fano variety of lines in the Grothendieck ring of varieties. The following is a version of this relation on the level of Chow motives:

Theorem 2.27 ([24]). Let $Y \subset \mathbb{P}^{n+1}(\mathbb{C})$ be a smooth cubic, and let $X=F(Y)$ be the Fano variety of lines in $Y$. There is an isomorphism of motives

$$
\Gamma: \quad h(X)(2) \oplus \bigoplus_{i=0}^{n} h(Y)(i) \cong h\left(Y^{[2]}\right) \text { in } \mathcal{M}_{\text {rat }} .
$$

Proof. This is [24, Theorem 5].

In particular, this implies the following:

Corollary 2.28. Let $Y \subset \mathbb{P}^{5}(\mathbb{C})$ be a smooth cubic fourfold, and let $X=F(Y)$ be the Fano variety of lines in $Y$. There exists a correspondence inducing an injection

$$
A_{\text {hom }}^{i}(X) \hookrightarrow A_{\text {hom }}^{i+2}(Y \times Y) \oplus \bigoplus A_{\text {hom }}^{3}(Y)
$$

Moreover, this can be done relatively: let $\mathcal{Y} \rightarrow B$ be a family of smooth cubic fourfolds, and let $\mathcal{X} \rightarrow B$ be the family of associated Fano varieties. Then there exists a relative correspondence $\Psi_{1} \in A^{6}\left(\mathcal{X} \times_{B}\left(\mathcal{Y} \times_{B} \mathcal{Y}\right)\right) \oplus \bigoplus A^{*}\left(\mathcal{X} \times_{B} \mathcal{Y}\right)$, inducing an injection

$$
\left(\left.\Psi_{1}\right|_{b}\right)_{*}: \quad A_{h o m}^{i}\left(X_{b}\right) \hookrightarrow A_{h o m}^{i+2}\left(Y_{b} \times Y_{b}\right) \oplus \bigoplus A_{h o m}^{3}\left(Y_{b}\right) \quad \forall b \in B .
$$

(Here $\left.\Psi_{1}\right|_{b}$ is shorthand for the restriction of $\Psi_{1}$ to the fibre $X_{b} \times\left(Y_{b}\right)^{2} \cup \cup\left(X_{b} \times Y_{b}\right)$.) 
Proof. The first statement follows immediately from theorem 2.27 , for it is readily seen that there is a correspondence-induced injection

$$
A^{i+2}\left(Y^{[2]}\right) \hookrightarrow A^{i+2}(Y \times Y) \oplus \bigoplus A^{*}(Y)
$$

Restricting to $A_{h o m}^{*}()$ (and remembering that $A_{h o m}^{i}(Y)=0$ for $i \neq 3$ ) gives the first statement.

To prove the "moreover" part, we need to understand how the correspondence $\Gamma$ of theorem 2.27 is constructed. The construction is based on the existence of a birational map (found by Galkin-Shinder [15, Proof of Theorem 5.1])

$$
\phi: \quad Y^{[2]} \rightarrow W,
$$

where $W \rightarrow Y$ denotes the $\mathbb{P}^{4}$-bundle whose fibre over $y \in Y$ is the set of lines in $\mathbb{P}^{5}$ passing through $y$. The indeterminacy locus $\tau: Z \hookrightarrow Y^{[2]}$ of $\phi$ is a $\mathbb{P}^{2}$-bundle $p: Z \rightarrow X$, and it turns out [48, Proposition 2.9] that the indeterminacy of $\phi$ is resolved by the blow-up with center $Z$. The map from $h(X)(2)$ to $h\left(Y^{[2]}\right)$ in theorem 3.1 is defined simply as

$$
\Gamma_{\tau} \circ{ }^{t} \Gamma_{p}: \quad h(X)(2) \rightarrow h\left(Y^{[2]}\right) \text { in } \mathcal{M}_{\text {rat }} .
$$

This construction naturally extends to the family: inside $\mathcal{Y}^{[2]}$ there is a codimension 2 locus $\tau: \mathcal{Z} \hookrightarrow \mathcal{Y}^{[2]}$ (defined as those points $x \in\left(Y_{b}\right)^{[2]}$ for which the line $L_{x}$ determined by $x$ is contained in $Y_{b}$ ), and $\mathcal{Z}$ has the structure of a $\mathbb{P}^{2}$-bundle $p: \mathcal{Z} \rightarrow \mathcal{X}$. One can thus define

$$
\Psi:=\Gamma_{\tau} \circ{ }^{t} \Gamma_{p} \in A^{6}\left(\mathcal{X} \times_{B} \mathcal{Y}^{[2]}\right) .
$$

The desired relative correspondence $\Psi_{1}$ is defined as the composition $\Psi_{1}=\Psi_{0} \circ \Psi$, where the correspondence

$$
\Psi_{0} \in A^{4}\left(\mathcal{Y}^{[2]} \times_{B}\left(\mathcal{Y} \times_{B} \mathcal{Y}\right)\right) \oplus \bigoplus A^{*}\left(\mathcal{Y}^{[2]} \times_{B} \mathcal{Y}\right)
$$

induces the fibrewise injection (4); this $\Psi_{0}$ obviously exists relatively.

\subsection{Cubics containing two planes.}

Notation 2.29. Let $P_{1}, P_{2} \subset \mathbb{P}^{5}(\mathbb{C})$ be two fixed disjoint planes, and consider smooth cubic fourfolds $Y_{b}$ containing these two planes. Such cubics form a family

$$
\mathcal{Y} \rightarrow B
$$

where $B \subset \bar{B} \subset \mathbb{P} H^{0}\left(\mathbb{P}^{5}, \mathcal{O}_{\mathbb{P}^{5}}(3)\right)$. Here $B$ is Zariski open in $\bar{B}$, and $\bar{B}$ is the closed subset parametrizing equations of the form

$$
f_{1}(u, v, w ; x, y, z)+f_{2}(u, v, w ; x, y, z)=0,
$$

where $f_{1}, f_{2}$ are bihomogeneous of bidegree $(1,2)$ resp. $(2,1)$ in the variables $[u, v, w]$ and $[x, y, z]$.

$$
\text { Let }
$$

$$
\mathcal{X} \rightarrow B
$$

be the universal family of Fano varieties of lines in cubic fourfolds containing two planes, i.e.

$$
\mathcal{X}:=\left\{(\ell, b) \mid \ell \subset Y_{b}\right\} \quad \subset G r(1,5) \times B
$$


(here $G r(1,5)$ is the Grassmannian of lines in $\left.\mathbb{P}^{5}\right)$. The fibre $X_{b}$ (of $\mathcal{X} \rightarrow B$ over $b \in B$ ) is the Fano variety of lines in the cubic $Y_{b}$.

Let

$$
\mathcal{S} \rightarrow B
$$

be the universal family of $K 3$ surfaces in $\mathbb{P}^{2} \times \mathbb{P}^{2}$ defined by the bihomogeneous equations

$$
f_{1}(u, v, w ; x, y, z)=f_{2}(u, v, w ; x, y, z)=0 .
$$

For later use, we record two lemmas that express a relation between the cubic $Y_{b}$ and the $K 3$ surface $S_{b}$ :

Lemma 2.30. Notation as in notation 2.29. There exist relative correspondences

$$
\Psi_{2} \in A^{3}\left(\mathcal{Y} \times_{B} \mathcal{S}\right), \quad \Xi_{2} \in A^{3}\left(\mathcal{S} \times_{B} \mathcal{Y}\right)
$$

with the property that

$$
\left(\left.\left(\Xi_{2} \circ \Psi_{2}\right)\right|_{Y_{b} \times Y_{b}}\right)_{*}=\mathrm{id}: \quad A_{h o m}^{3}\left(Y_{b}\right) \rightarrow A_{h o m}^{3}\left(Y_{b}\right) \quad \forall b \in B .
$$

Proof. The proof of the rationality of the cubics $Y_{b}$ (cf. for instance [16, Section 5], [17, Section 1.2]) can be done by showing that for any $b \in B$ there exists a birational map

$$
\rho_{b}: \quad \mathbb{P}^{2} \times \mathbb{P}^{2} \rightarrow Y_{b} .
$$

Taking coordinates $[u, v, w],[x, y, z]$ for $\mathbb{P}^{2} \times \mathbb{P}^{2}$, the indeterminacy locus of $\rho_{b}$ is exactly the $K 3$ surface $S_{b}$, and the blow-up $Z_{b}$ of $\mathbb{P}^{2} \times \mathbb{P}^{2}$ along $S_{b}$ admits a morphism $\widetilde{\rho}_{b}: Z_{b} \rightarrow Y_{b}$ resolving the indeterminacy of $\rho_{b}$. Let $i_{b}: E_{b} \hookrightarrow Z_{b}$ denote the exceptional divisor of the blow-up, and let $p_{b}: E_{b} \rightarrow S_{b}$ denote the induced morphism. The usual blow-up exact sequence implies that the composition

$$
A_{\text {hom }}^{3}\left(Y_{b}\right) \stackrel{\left(\widetilde{\rho_{b}}\right)^{*}}{\longrightarrow} A_{\text {hom }}^{3}\left(Z_{b}\right) \stackrel{\left(i_{b}\right)^{*}}{\longrightarrow} A_{h o m}^{3}\left(E_{b}\right) \stackrel{\left(p_{b}\right)_{*}}{\longrightarrow} A_{h o m}^{2}\left(S_{b}\right)
$$

is injective, and a left-inverse is given by

$$
A_{\text {hom }}^{2}\left(S_{b}\right) \stackrel{\left(p_{b}\right)^{*}}{\longrightarrow} A_{\text {hom }}^{2}\left(E_{b}\right) \stackrel{\left(i_{b}\right)_{*}}{\longrightarrow} A_{\text {hom }}^{3}\left(Z_{b}\right) \stackrel{\left(\widetilde{\rho_{b}}\right)_{*}}{\longrightarrow} A_{\text {hom }}^{3}\left(Y_{b}\right) .
$$

This defines fibrewise correspondences between $Y_{b}$ and $S_{b}$. By looking at the blow-up of $\mathbb{P}^{2} \times$ $\mathbb{P}^{2} \times B$ along $\mathcal{S}$, one sees that these correspondences exist relatively, as desired.

Lemma 2.31. Notation as in notation 2.29. There exists a relative correspondence

$$
\Psi_{3} \in A^{6}\left(\left(\mathcal{Y} \times_{B} \mathcal{Y}\right) \times_{B}\left(\mathcal{S} \times_{B} \mathcal{S}\right)\right) \oplus A^{*}\left(\left(\mathcal{Y} \times_{B} \mathcal{Y}\right) \times_{B} \mathcal{S}\right)^{\oplus 2}
$$

with the property that

$$
\left(\left.\Psi_{3}\right|_{b}\right)_{*}: \quad A_{\text {hom }}^{4}\left(Y_{b} \times Y_{b}\right) \rightarrow A_{h o m}^{2}\left(S_{b} \times S_{b}\right) \oplus A_{h o m}^{2}\left(S_{b}\right)^{\oplus 2}
$$

is injective for very general $b \in B$.

Proof. Lemma 2.30 implies that the map of Chow motives

$$
\left(\Psi_{2}, \Psi^{\prime}\right): \quad h\left(Y_{b}\right) \rightarrow h\left(S_{b}\right)(1) \oplus \bigoplus_{i=0}^{4} \mathbb{L}(i) \quad \text { in } \mathcal{M}_{\text {rat }}
$$


admits a left-inverse (i.e. the left-hand side is a direct summand of the right-hand side). This implies that there is an induced map

$$
h\left(Y_{b} \times Y_{b}\right) \rightarrow h\left(S_{b} \times S_{b}\right)(2) \oplus \bigoplus_{j} h\left(S_{b}\right)(j) \oplus \bigoplus_{k} \mathbb{L}(k) \text { in } \mathcal{M}_{\text {rat }}
$$

admitting a left-inverse. In particular, taking $A_{h o m}^{4}()$ on both sides, we find there is an injection

$$
A_{\text {hom }}^{4}\left(Y_{b} \times Y_{b}\right) \hookrightarrow A_{h o m}^{2}\left(S_{b} \times S_{b}\right) \oplus \bigoplus A_{h o m}^{2}\left(S_{b}\right)
$$

which is induced by various combinations of $\Psi_{2}$ and $\Psi^{\prime}$. We have seen (lemma 2.30) that $\Psi_{2}$ exists relatively. As for $\Psi^{\prime}$, this is obtained by considering the "trivial" cycles $h^{i} \in A^{i}\left(Y_{b}\right)$ coming from a hyperplane section $h \in A^{1}\left(\mathbb{P}^{5}\right)$, plus choosing a basis for $N^{2} H^{4}\left(Y_{b}\right)$. The cycles $h^{i}$ obviously exist relatively. Let $B_{0} \subset B$ be the locus where $\operatorname{dim} N^{2} H^{4}\left(Y_{b}\right)$ equals 3 ; this $B_{0}$ is the intersection of a countable number of Zariski opens in $B$. The correspondence $\Psi^{\prime}$ exists as a relative correspondence over $B_{0}$. It follows there exists a relative correspondence $\Psi_{3}$ as indicated.

Remark 2.32. Let $Y_{b}$ be a smooth cubic fourfold containing two planes. We will say that $S_{b}$ is the related K3 surface. (We want to avoid the terminology "associated K3 surface", which already has a different meaning [16], [17].)

\subsection{The generalized Franchetta conjecture for certain $K 3$ surfaces.}

Proposition 2.33. Let $\mathcal{S} \rightarrow B$ be the family of $K 3$ surfaces of notation 2.29. Let $\Gamma \in A^{2}(\mathcal{S})$ be a relative cycle such that

$$
\left.\Gamma\right|_{S_{b}}=0 \text { in } H^{4}\left(S_{b}\right) \quad \forall b \in B
$$

Then also

$$
\left.\Gamma\right|_{S_{b}}=0 \text { in } A^{2}\left(S_{b}\right) \quad \forall b \in B .
$$

Proof. Let $\mathbb{P}:=\mathbb{P}^{2} \times \mathbb{P}^{2}$, and let $U \rightarrow \mathbb{P}$ be the rank 2 vector bundle

$$
U:=\mathcal{O}_{\mathbb{P}}(1,2) \oplus \mathcal{O}_{\mathbb{P}}(2,1) .
$$

Let

$$
\bar{B}:=\mathbb{P} H^{0}(\mathbb{P}, U), \quad \overline{\mathcal{S}}:=\{(x, f) \mid f(x)=0\} \quad \subset \mathbb{P} \times \bar{B} .
$$

Then $B$ and $\mathcal{S}$ are Zariski opens in $\bar{B}$ resp. $\overline{\mathcal{S}}$. There is a diagram

$$
\begin{array}{lll}
\overline{\mathcal{S}} & \stackrel{\nu}{\rightarrow} & \mathbb{P} \\
\downarrow^{\downarrow} \pi & &
\end{array}
$$

Let $\phi_{b}: S_{b} \hookrightarrow \mathcal{S} \hookrightarrow \overline{\mathcal{S}}$ and $i_{b}: S_{b} \hookrightarrow \mathbb{P}$ denote the inclusion morphisms. We claim that there is equality

$$
\operatorname{Im}\left(A^{2}(\overline{\mathcal{S}}) \stackrel{\left(\phi_{b}\right)^{*}}{\longrightarrow} A^{2}\left(S_{b}\right)\right)=\operatorname{Im}\left(A^{2}(\mathbb{P}) \stackrel{\left(i_{b}\right)^{*}}{\longrightarrow} A^{2}\left(S_{b}\right)\right) .
$$

This claim implies the proposition: indeed,

$$
A^{2}(\mathbb{P})=A^{2}\left(\mathbb{P}^{2} \times \mathbb{P}^{2}\right)=\bigoplus_{k+\ell=2} A^{k}\left(\mathbb{P}^{2}\right) \otimes A^{\ell}\left(\mathbb{P}^{2}\right)
$$


is a 3-dimensional $\mathbb{Q}$-vector space with generators $h^{2} \times \mathbb{P}^{2}, h \times h, \mathbb{P}^{2} \times h^{2}$ (where $h \in A^{1}\left(\mathbb{P}^{2}\right.$ ) is the class of a hyperplane section). Thus every element in $A^{2}(\mathbb{P})$ is a sum of intersections of divisors. Since intersections of two divisors are a multiple of the distinguished class $\mathfrak{o}_{S_{b}}$ in $A^{2}\left(S_{b}\right)$ [4], the claim (applied to any lifting $\bar{\Gamma}$ of $\Gamma$ to $A^{2}(\overline{\mathcal{S}})$ ) implies that

$$
\left.\Gamma\right|_{S_{b}}=\left(\phi_{b}\right)^{*}(\bar{\Gamma})=m_{b} \mathfrak{o}_{S_{b}} \text { in } A^{2}\left(S_{b}\right)
$$

for some $m_{b} \in \mathbb{Q}$, and the proposition is proven.

To prove the claim, we apply the argument of [33, Lemma 2.1] (where (5) is proven in a very similar set-up). First, one observes that $U \rightarrow \mathbb{P}$ is globally generated and so $\nu: \overline{\mathcal{S}} \rightarrow \mathbb{P}$ is a projective bundle; the fibre over a point $x \in \mathbb{P}$ is

$$
\mathbb{P} H^{0}\left(\mathbb{P}, U \otimes I_{x}\right)
$$

(where $I_{x}$ denotes the ideal sheaf of $x$ ). The projective bundle formula gives an equality

$$
A^{r}(\overline{\mathcal{S}})=\xi^{r} \cdot \nu^{*} A^{0}(\mathbb{P})+\xi^{r-1} \cdot \nu^{*} A^{1}(\mathbb{P})+\cdots+\nu^{*} A^{r}(\mathbb{P}),
$$

where $\xi \in A^{1}(\overline{\mathcal{S}})$ denotes the relative hyperplane class. In particular, if $H \in A^{1}(\bar{B})$ denotes the hyperplane class, we can write

$$
\pi^{*}(H)=a \cdot \xi+\nu^{*}(b) \text { in } A^{1}(\overline{\mathcal{S}})
$$

for some $a \in \mathbb{Q}$ and $b \in A^{1}(\mathbb{P})$. The constant $a$ must be non-zero, for otherwise

$$
\pi^{*}\left(H^{\operatorname{dim} \bar{B}}\right)=\nu^{*}\left(b^{\operatorname{dim} \bar{B}}\right) \text { in } A^{\operatorname{dim} \bar{B}}(\overline{\mathcal{S}}),
$$

which is absurd (the right-hand side is 0 since $\operatorname{dim} \bar{B}>4=\operatorname{dim} \mathbb{P}$, but the left-hand side is the pullback of an effective $0-$ cycle and is non-zero).

It follows that one may write

$$
\xi=\frac{1}{a}\left(\pi^{*}(H)-\nu^{*}(b)\right) \text { in } A^{1}(\overline{\mathcal{S}}) .
$$

Plugging this in equality (6) and taking $r=2$, we find that

$$
A^{2}(\overline{\mathcal{S}})=\left(\pi^{*}(H)-\nu^{*}(b)\right)^{2} \cdot \nu^{*} A^{0}(\mathbb{P}) \oplus\left(\pi^{*}(H)-\nu^{*}(b)\right) \cdot \nu^{*} A^{1}(\mathbb{P}) \oplus \nu^{*} A^{2}(\mathbb{P}) .
$$

The class $\pi^{*}(H)$ vanishes when restricting to $S_{b}$, i.e. $\left(\phi_{b}\right)^{*} \pi^{*}(H)=0$ in $A^{2}\left(S_{b}\right)$ for any $b \in B$, and so

$$
\begin{aligned}
\operatorname{Im}\left(A^{2}(\overline{\mathcal{S}}) \stackrel{\left(\phi_{b}\right)^{*}}{\longrightarrow} A^{2}\left(S_{b}\right)\right) & =\operatorname{Im}\left(\left(\nu^{*}\left(b^{2}\right) \cdot \nu^{*} A^{0}(\mathbb{P}) \oplus \nu^{*}(b) \cdot \nu^{*} A^{1}(\mathbb{P}) \oplus \nu^{*} A^{2}(\mathbb{P})\right) \stackrel{\left(\phi_{b}\right)^{*}}{\longrightarrow} A^{2}\left(S_{b}\right)\right) \\
& =\operatorname{Im}\left(\nu^{*} A^{2}(\mathbb{P}) \stackrel{\left(\phi_{b}\right)^{*}}{\longrightarrow} A^{2}\left(S_{b}\right)\right) \\
& =\operatorname{Im}\left(A^{2}(\mathbb{P}) \stackrel{\left(i_{b}\right)^{*}}{\longrightarrow} A^{2}\left(S_{b}\right)\right)
\end{aligned}
$$

proving the claim.

Remark 2.34. For any $b \in B$, let $\mathfrak{o}_{S_{b}} \in A^{2}\left(S_{b}\right)$ denote the distinguished $0-$ cycle of [4]. An equivalent way of stating proposition 2.33 is as follows: given any relative cycle $\Gamma \in A^{2}(\mathcal{S})$, the restriction

is a multiple of $\mathfrak{o}_{S_{b}}$ in $A^{2}\left(S_{b}\right)$.

$$
\left.\Gamma\right|_{S_{b}} \in A^{2}\left(S_{b}\right)
$$


This statement is very similar to the "generalized Franchetta conjecture" for K3 surfaces, which was formulated by O'Grady [32, Section 5] and proven in certain cases by Pavic-ShenYin [33].

\section{MAIN RESULTS}

This section proves the two main results of this note, theorems 3.1 and 3.2. The proofs are based on the method of "spread" of cycles in nice families, as developed by Voisin [43], [44], [45], [46].

The first main result is a "generalized Franchetta conjecture" type of statement for certain Hilbert squares:

Theorem 3.1. Let $g \in\{2,3,4,5,6,8\}$. Let $\mathcal{X} \rightarrow B$ denote the universal family of Hilbert squares of genus $g$ K3 surfaces (notation 2.24). Let $\Gamma \subset \mathcal{X}$ be a codimension 2 subvariety. Then the restriction $\left.\Gamma\right|_{X_{b}} \in A^{2}\left(X_{b}\right)$ is in $A_{(0)}^{2}\left(X_{b}\right)$ for all $b \in B$.

Proof. Let us fix an integer $g \in\{2,3,4,5,6,8\}$, and write

$$
\mathcal{S} \rightarrow B, \quad \mathcal{X}:=\mathcal{S}^{[2]} \rightarrow B
$$

for the universal family of genus $g K 3$ surfaces (i.e., the family $\mathcal{S}_{g} \rightarrow B_{g}$ of subsection 2.8), resp. the universal Hilbert square of a genus $g K 3$ surface (as in notation 2.24).

One knows (remark 2.11) that the MCK decomposition $\left\{\Pi_{j}^{X_{b}}\right\}$ for the $X_{b}$ of [38] exists relatively, in particular there exists a relative correspondence $\Pi_{2}^{\mathcal{X}} \in A^{4}\left(\mathcal{X} \times_{B} \mathcal{X}\right)$ such that

$$
\left.\Pi_{2}^{\mathcal{X}}\right|_{X_{b} \times X_{b}}=\Pi_{2}^{X_{b}} \quad \text { in } A^{4}\left(X_{b} \times X_{b}\right) \quad \forall b \in B .
$$

Let us now consider the relative cycle

$$
\Gamma_{0}:=\left(\Pi_{2}^{\mathcal{X}}\right)_{*}(\Gamma) \quad \in A^{2}(\mathcal{X}) .
$$

We know this is fibrewise homologically trivial:

$$
\left.\Gamma_{0}\right|_{X_{b}}=0 \text { in } H^{4}\left(X_{b}\right) \quad \forall b \in B .
$$

(This is simply because $\Pi_{2}^{X_{b}} \circ \Pi_{4}^{X_{b}}=0$ in $H^{8}\left(X_{b} \times X_{b}\right)$.)

What we desire to prove (in order to establish the theorem) is that there is a fibrewise rational equivalence

$$
\left.\Gamma_{0}\right|_{X_{b}} \stackrel{? ?}{=} 0 \text { in } A^{2}\left(X_{b}\right) \quad \forall b \in B .
$$

It is easier to move things to the family $\mathcal{S} \times{ }_{B} \mathcal{S}$. To this end, let

$$
\Psi \in A^{4}\left(\mathcal{X} \times_{B}\left(\mathcal{S} \times_{B} \mathcal{S}\right)\right)
$$

be the relative correspondence defined by the standard diagram

$$
\begin{array}{cc}
\mathcal{X}:=\mathcal{S}^{[2]} & \leftarrow \widetilde{\mathcal{S} \times{ }_{B} \mathcal{S}} \\
\downarrow & \downarrow \\
\mathcal{S}^{(2)}:=\left(\mathcal{S} \times{ }_{B} \mathcal{S}\right) / \mathfrak{S}_{2} & \leftarrow \mathcal{S} \times_{B} \mathcal{S}
\end{array}
$$


Let us consider the relative correspondence

$$
\Gamma_{1}:=(\Psi)_{*}\left(\Gamma_{0}\right) \quad \in A^{2}\left(\mathcal{S} \times_{B} \mathcal{S}\right) .
$$

We recall from equality (7) that $\left.\Gamma_{0}\right|_{X_{b}}$ is in $A_{h o m}^{2}\left(X_{b}\right)=A_{A J}^{2}\left(X_{b}\right)$ for all $b \in B$. The restriction $\Psi_{b}:=\left.\Psi\right|_{X_{b} \times\left(S_{b}\right)^{2}}$ acts as

$$
\left(\Psi_{b}\right)_{*}: \quad A_{A J}^{2}\left(X_{b}\right) \rightarrow A_{A J}^{2}\left(\widetilde{S_{b} \times S_{b}}\right) \rightarrow A_{A J}^{2}\left(S_{b} \times S_{b}\right),
$$

where the first arrow is an injection and the second arrow is an isomorphism. Hence, in order to prove (8) it will suffice to prove

$$
\left.\Gamma_{1}\right|_{X_{b}} \stackrel{? ?}{=} 0 \quad \text { in } A^{2}\left(S_{b} \times S_{b}\right) \quad \forall b \in B
$$

But the family $\mathcal{S} \rightarrow B$ enters into the set-up of proposition 2.22 (with $M=\mathbb{P}_{g}$ and $L_{j}$ as given in subsection 2.8), and (7) implies that the cycle $\Gamma_{1} \in A^{2}\left(\mathcal{S} \times{ }_{B} \mathcal{S}\right)$ is fibrewise homologically trivial:

$$
\left.\Gamma_{1}\right|_{S_{b} \times S_{b}}=0 \text { in } H^{4}\left(S_{b} \times S_{b}\right) \quad \forall b \in B .
$$

Proposition 2.22 allows us to conclude that there exists $\delta \in A^{2}\left(\mathbb{P}_{g} \times \mathbb{P}_{g}\right)$ such that there is fibrewise rational equivalence

$$
\left.\Gamma_{1}\right|_{S_{b} \times S_{b}}=\left.\delta\right|_{S_{b} \times S_{b}} \quad \text { in } A^{2}\left(S_{b} \times S_{b}\right) \quad \forall b \in B .
$$

Let us decompose

$$
\delta=\delta_{0}+\delta_{1}+\delta_{2} \quad \in A^{2}\left(\mathbb{P}_{g}\right) \otimes A^{0}\left(\mathbb{P}_{g}\right) \oplus A^{1}\left(\mathbb{P}_{g}\right) \otimes A^{1}\left(\mathbb{P}_{g}\right) \oplus A^{0}\left(\mathbb{P}_{g}\right) \otimes A^{2}\left(\mathbb{P}_{g}\right),
$$

where it is understood that $\delta_{0}$ is in the first summand, and $\delta_{1}, \delta_{2}$ are in the second resp. last summand. (This decomposition is possible because $\mathbb{P}_{g}$ has trivial Chow groups, and so

$$
\left.A^{*}\left(\mathbb{P}_{g} \times \mathbb{P}_{g}\right)=A^{*}\left(\mathbb{P}_{g}\right) \otimes A^{*}\left(\mathbb{P}_{g}\right) .\right)
$$

As the restriction $\left.\delta\right|_{S_{b} \times S_{b}}$ is homologically trivial (for each $b \in B$ ), and the $\left.\delta_{j}\right|_{S_{b} \times S_{b}}$ land in different summands of the Künneth decomposition of cohomology, we have

$$
\left.\delta_{j}\right|_{S_{b} \times S_{b}} \in A_{\text {hom }}^{2}\left(S_{b} \times S_{b}\right) \quad \forall b \in B, j=0,1,2 .
$$

From this, we may conclude that

$$
\left.\delta_{1}\right|_{S_{b} \times S_{b}}=0 \text { in } A^{2}\left(S_{b} \times S_{b}\right) \quad \forall b \in B,
$$

thanks to Voisin's proof of the Beauville-Voisin conjecture for $\left(S_{b}\right)^{m}$ with $m$ small [42, Proposition 2.2].

As for the component $\delta_{0}$, let us take a relatively ample divisor $h \in A^{1}(\mathcal{S})$ (e.g., induced by an ample divisor on $\mathbb{P}_{g}$ ), and consider the relative cycle

$$
\delta_{00}:=\left(p_{1}\right)_{*}\left(\left(\left.\left(\delta_{0} \times B\right)\right|_{\mathcal{S} \times{ }_{B} \mathcal{S}}\right) \cdot\left(p_{2}\right)^{*}\left(h^{2}\right)\right) \in A^{2}(\mathcal{S}),
$$

where $p_{i}: \mathcal{S} \times{ }_{B} \mathcal{S} \rightarrow \mathcal{S}$ for $i=1,2$ denotes projection on the first resp. second factor. We have that

$$
\left.\delta_{0}\right|_{S_{b} \times S_{b}}=d \cdot\left(p_{1}\right)^{*}\left(\left.\delta_{00}\right|_{S_{b}}\right) \quad \text { in } A^{2}\left(S_{b} \times S_{b}\right) \quad \forall b \in B,
$$


for some non-zero integer $d$. We observe that (11) implies that the restriction $\left.\delta_{00}\right|_{S_{b}}$ is of degree 0 for all $b \in B$. But then, reasoning exactly as in [33], we must have that $\left.\delta_{00}\right|_{S_{b}}$ is rationally trivial. (More in detail: by construction, one has

$$
\left.\delta_{00}\right|_{S_{b}} \in \operatorname{Im}\left(A^{2}\left(\mathbb{P}_{g}\right) \rightarrow A^{2}\left(S_{b}\right)\right) .
$$

However, it is known that

$$
\operatorname{Im}\left(A^{2}\left(\mathbb{P}_{g}\right) \rightarrow A^{2}\left(S_{b}\right)\right)=\mathbb{Q}\left[\mathfrak{o}_{S_{b}}\right]
$$

(where $\mathfrak{o}_{S_{b}}$ is the distinguished point of [4]); Pavic-Shen-Yin supply not one but two proofs for this [33, Proposition 3.1].)

It follows that also

$$
\left.\delta_{0}\right|_{S_{b} \times S_{b}}=0 \text { in } A^{2}\left(S_{b} \times S_{b}\right) \quad \forall b \in B .
$$

The proof for $\delta_{2}$ is the same as for $\delta_{0}$. We conclude that

$$
\left.\delta\right|_{S_{b} \times S_{b}}=0 \text { in } A^{2}\left(S_{b} \times S_{b}\right) \quad \forall b \in B,
$$

and so (10) gives the desired equality (9).

We now come to the second main result of this note:

Theorem 3.2. Let $g \in\{2,3,4,5,6,8\}$. Let $\mathcal{X} \rightarrow B$ denote the universal family of Hilbert squares of genus $g$ K 3 surfaces (notation 2.24). Let $\Gamma \subset \mathcal{X}$ be a codimension 2 subvariety. Assume the restriction

$$
\Gamma_{b}:=\left.\Gamma\right|_{X_{b}} \in A^{2}\left(X_{b}\right)
$$

is Lagrangian (i.e., $\cup \Gamma_{b}: H^{2,0}\left(X_{b}\right) \rightarrow H^{4,2}\left(X_{b}\right)$ is the zero map), for very general $b \in B$. Then

$$
\cdot \Gamma_{b}: \quad A_{h o m}^{2}\left(X_{b}\right) \rightarrow A^{4}\left(X_{b}\right)
$$

is the zero map, for all $b \in B$.

Proof. Thanks to theorem 2.1, there is a splitting

$$
A_{\text {hom }}^{2}\left(X_{b}\right)=A_{(2)}^{2}\left(X_{b}\right) \oplus\left(A_{(0)}^{2}\left(X_{b}\right) \cap A_{h o m}^{2}\left(X_{b}\right)\right),
$$

where the second summand is conjecturally zero. It is known that the intersection product map

$$
A_{(0)}^{2}\left(X_{b}\right) \otimes\left(A_{(0)}^{2}\left(X_{b}\right) \cap A_{h o m}^{2}\left(X_{b}\right)\right) \rightarrow A^{4}\left(X_{b}\right)
$$

is the zero map [38, Theorem 3]. Since $\Gamma_{b} \in A_{(0)}^{2}\left(X_{b}\right)$ by theorem 3.1, we are reduced to proving that

is the zero map.

$$
\cdot \Gamma_{b}: \quad A_{(2)}^{2}\left(X_{b}\right) \rightarrow A^{4}\left(X_{b}\right)
$$

Let us consider a resolution of singularities $f: \widetilde{\Gamma} \rightarrow \Gamma$, and let $\tau: \widetilde{\Gamma} \hookrightarrow \mathcal{X}$ denote the composition of $f$ with the inclusion morphism $\Gamma \hookrightarrow \mathcal{X}$. Let us consider the relative correspondence

$$
\Gamma_{0}:=\mathcal{L} \circ \Gamma_{\tau} \circ{ }^{t} \Gamma_{\tau} \circ \Pi_{2}^{\mathcal{X}} \in A^{4}\left(\mathcal{X} \times{ }_{B} \mathcal{X}\right),
$$

where $\Pi_{2}^{\mathcal{X}}$ and $\mathcal{L}$ are as in remark 2.11 resp. lemma 2.18 .

By construction, for any $b \in B$, the restriction $\left.\Gamma_{0}\right|_{X_{b} \times X_{b}}$ acts on Chow groups as

$$
A^{i}\left(X_{b}\right) \rightarrow\left(\Pi_{2}^{X_{b}}\right)_{*} A^{i}\left(X_{b}\right) \stackrel{\Gamma_{b}}{\longrightarrow} A^{i+2}\left(X_{b}\right) \stackrel{\left(L_{b}\right)_{*}}{\longrightarrow} A^{i}\left(X_{b}\right) .
$$


In particular, taking $i=2$ and applying the fact that $\Gamma_{b} \in A_{(0)}^{2}\left(X_{b}\right)$ (theorem 3.1), we get an action

$$
\left(\left.\Gamma_{0}\right|_{X_{b} \times X_{b}}\right)_{*}: \quad A_{(2)}^{2}\left(X_{b}\right) \stackrel{\text { id }}{\rightarrow} A_{(2)}^{2}\left(X_{b}\right) \stackrel{\Gamma_{b}}{\longrightarrow} A_{(2)}^{4}\left(X_{b}\right) \stackrel{\left(L_{b}\right)_{*}}{\longrightarrow} A_{(2)}^{2}\left(X_{b}\right) .
$$

We know that the last arrow is an isomorphism (theorem 2.17). Also, $\Pi_{2}^{X_{b}}$ acts as the identity on $A_{(2)}^{2}\left(X_{b}\right)$. Thus, to prove theorem 3.2 we are reduced to proving that

$$
\left(\left.\left(\Pi_{2}^{\mathcal{X}} \circ \Gamma_{0}\right)\right|_{X_{b} \times X_{b}}\right) * \stackrel{? ?}{=} 0: \quad A_{(2)}^{2}\left(X_{b}\right) \rightarrow A_{(2)}^{2}\left(X_{b}\right) \quad \forall b \in B .
$$

The input we have at our disposition is that we know (from the coisotropic assumption) that

$$
\left(\left.\Gamma_{0}\right|_{X_{b} \times X_{b}}\right)_{*}=0: \quad H^{2,0}\left(X_{b}\right) \rightarrow H^{2,0}\left(X_{b}\right) \text { for very general } b \in B .
$$

We observe that (13), combined with the Lefschetz $(1,1)$ theorem, implies the following: for very general $b \in B$, there exist a curve $C_{b} \subset X_{b}$, a divisor $D_{b}$ and a cycle $\gamma_{b}$ supported on $C_{b} \times D_{b} \subset X_{b} \times X_{b}$, such that

$$
\left.\Gamma_{0}\right|_{X_{b} \times X_{b}}-\gamma_{b}=0 \text { in } H^{8}\left(X_{b} \times X_{b}\right) .
$$

Thanks to Voisin's key result [43, Proposition 3.7] (cf. also [44, Proposition 4.25]), it is possible to spread out these data. That is, there exist subvarieties $\mathcal{C} \subset \mathcal{X}, \mathcal{D} \subset \mathcal{X}$ of codimension 3 resp. 1 , and a cycle $\gamma \in A^{4}\left(\mathcal{X} \times_{B} \mathcal{X}\right)$ supported on $\mathcal{C} \times_{B} \mathcal{D}$ that does the job of the various $\gamma_{b}$, i.e. such that

$$
\left.\left(\Gamma_{0}-\gamma\right)\right|_{X_{b} \times X_{b}}=0 \text { in } H^{8}\left(X_{b} \times X_{b}\right) \text { for very general } b \in B \text {. }
$$

In other words, the relative correspondence defined as

$$
\Gamma_{1}:=\Gamma_{0}-\gamma \quad \in A^{4}\left(\mathcal{X} \times_{B} \mathcal{X}\right)
$$

has the property that

$$
\left.\Gamma_{1}\right|_{X_{b} \times X_{b}}=0 \text { in } H^{8}\left(X_{b} \times X_{b}\right) \text { for very general } b \in B .
$$

It is more convenient to move to correspondences living in $A^{2}\left(\mathcal{S} \times{ }_{B} \mathcal{S}\right)$, where $\mathcal{S} \rightarrow B$ is (as before) the family of $K 3$ surfaces of genus $g$. That is, first we consider the relative correspondence

$$
\Gamma_{2}:=\Psi \circ \Gamma_{1} \circ{ }^{t} \Psi \quad \in A^{4}\left(\mathcal{S}^{2 / B} \times_{B} \mathcal{S}^{2 / B}\right),
$$

where $\Psi \in A^{4}\left(\mathcal{X} \times{ }_{B} \mathcal{S}^{2 / B}\right)$ is as in the proof of theorem 3.1. Next, we define relative correspondences

$$
\Gamma_{3}^{i, j}:=\Xi_{i} \circ \Gamma_{2} \circ \Theta_{j} \quad \in A^{2}\left(\mathcal{S} \times_{B} \mathcal{S}\right) \quad(1 \leq i, j \leq 2),
$$

where $\Xi_{i}, \Theta_{j}$ are as in proposition 2.13. The relative correspondence $\Gamma_{1}$ being fibrewise homologically trivial (equation (14)), the same holds for $\Gamma_{2}$ and $\Gamma_{3}^{i, j}$ :

$$
\left.\Gamma_{3}^{i, j}\right|_{S_{b} \times S_{b}}=0 \text { in } H^{4}\left(S_{b} \times S_{b}\right) \text { for very general } b \in B \quad(1 \leq i, j \leq 2) .
$$

We can now apply proposition 2.22 to the $\Gamma_{3}^{i, j}$ (with $M=\mathbb{P}_{g}$ and $L_{r}$ as given in subsection 2.8). The conclusion is that there is a fibrewise rational equivalence

$$
\left.\Gamma_{3}^{i, j}\right|_{S_{b} \times S_{b}}=0 \quad \text { in } A^{2}\left(S_{b} \times S_{b}\right) \quad \forall b \in B \quad(1 \leq i, j \leq 2) .
$$


In particular, this implies that

$$
\Pi_{2}^{\mathcal{S}^{2 / B}} \circ \Gamma_{2} \circ \Pi_{2}^{\mathcal{S}^{2 / B}}=\sum_{i, j \in\{1,2\}} \Theta_{i} \circ \Gamma_{3}^{i, j} \circ \Xi_{j} \in A^{4}\left(\mathcal{S}^{2 / B} \times{ }_{B} \mathcal{S}^{2 / B}\right)
$$

is fibrewise rationally trivial (the equality follows from proposition 2.13). Composing some more, we find that also

$$
{ }^{t} \Psi \circ \Pi_{2}^{\mathcal{S}^{2 / B}} \circ \Gamma_{2} \circ \Pi_{2}^{\mathcal{S}^{2 / B}} \circ \Psi \quad \in A^{4}\left(\mathcal{X} \times{ }_{B} \mathcal{X}\right)
$$

is fibrewise rationally trivial. In view of the definition of $\Gamma_{2}$ (plus the fact that $\left(\Psi_{b}\right)^{*}\left(\Psi_{b}\right)_{*}$ is a multiple of the identity on $A_{h o m}^{2}\left(X_{b}\right)$ ), this implies that

$$
\left.\left.\left(\Pi_{2}^{\mathcal{X}} \circ \Gamma_{1} \circ \Pi_{2}^{\mathcal{X}}\right)\right|_{X_{b} \times X_{b}}\right)_{*}=0: \quad A_{\text {hom }}^{2}\left(X_{b}\right) \rightarrow A^{2}\left(X_{b}\right) \quad \forall b \in B .
$$

As $A_{(2)}^{2}\left(X_{b}\right) \subset A_{h o m}^{2}\left(X_{b}\right)$, this implies in particular that

$$
A_{(2)}^{2}\left(X_{b}\right) \stackrel{\text { id }}{\rightarrow} A_{(2)}^{2}\left(X_{b}\right) \stackrel{\left(\left.\Gamma_{1}\right|_{X_{b} \times X_{b}}\right)_{*}}{\longrightarrow} A^{2}\left(X_{b}\right) \stackrel{\left(\Pi_{2}^{X_{b}}\right)_{*}}{\longrightarrow} A_{(2)}^{2}\left(X_{b}\right)
$$

is the zero map, for all $b \in B$.

For general $b \in B$, the restriction of the cycle $\delta$ to the fibre $X_{b} \times X_{b}$ will be supported on (curve) $\times\left(\right.$ divisor), and so will act trivially on $A^{2}\left(X_{b}\right)$ for dimension reasons. That is, for general $b \in B$ we have equality

$$
\left(\left.\Gamma_{1}\right|_{X_{b} \times X_{b}}\right)_{*}=\left(\left.\Gamma_{0}\right|_{X_{b} \times X_{b}}\right)_{*}: \quad A^{2}\left(X_{b}\right) \rightarrow A^{2}\left(X_{b}\right) .
$$

The above thus implies that

$$
A_{(2)}^{2}\left(X_{b}\right) \stackrel{\left(\left.\Gamma_{0}\right|_{X_{b} \times X_{b}}\right)_{*}}{\longrightarrow} A^{2}\left(X_{b}\right) \stackrel{\left(\Pi_{2}^{X_{b}}\right)_{*}}{\longrightarrow} A_{(2)}^{2}\left(X_{b}\right)
$$

is the zero map, for general $b \in B$. That is, we have proven the desired statement (12) for general $b \in B$.

To extend the statement to all $b \in B$, one notes that the construction of [43, Proposition 3.7] (which was used above to globalize the various $\gamma_{b}$ ) can be done locally around a given $b_{0} \in B$.

Remark 3.3. Huybrechts ventures the following guess: "Any distinguished curve, i.e. a curve that is naturally defined in all generic K3 surfaces of fixed degree, should be a constant cycle curve" [19, Section 8.3 Footnote 5].

Similarly, in the set-up of theorem 3.1, could it be the case that for any codimension 2 subvariety $\Gamma \subset \mathcal{X}$ flat over $B$, the restriction $\left.\Gamma\right|_{X_{b}}$ is a constant cycle surface provided it is irreducible

? This would somehow "explain" that the restriction $\left.\Gamma\right|_{X_{b}}$ is in $A_{(0)}^{2}\left(X_{b}\right)$, in view of conjecture 1.2.

Remark 3.4. Here is a slightly different way of proving theorem 3.2: Let $X_{b}$ be the Hilbert square of a very general $K 3$ surface $S_{b}$ of genus $g \in\{2,3,4,5,6,8\}$. Let $\Gamma_{b} \subset X_{b}$ be a subvariety of codimension 2 that is Lagrangian. It follows from a result of Voisin [47, Proposition 4.2] that there is a homological equivalence

$$
\Gamma_{b}=\sum_{i} \lambda_{i} C_{b}^{i} \text { in } H^{4}\left(X_{b}\right),
$$


where $\lambda_{i} \in \mathbb{Q}$ and $C_{b}^{i} \subset X_{b}$ is a constant cycle surface.

Suppose we know in addition that $\Gamma_{b} \in A_{(0)}^{2}\left(X_{b}\right)$ is the restriction of a relative cycle $\Gamma \in$ $A^{2}(\mathcal{X})$ as in theorem 3.2). Then, one may "spread out" the fibrewise surfaces $C_{b}^{i}$ and use the argument of theorem 3.1 (or alternatively, the more recent [12, Theorems 1.4 and 1.5]) to conclude that there is a rational equivalence

$$
\Gamma_{b}=\sum_{i} \lambda_{i} C_{i} \text { in } A^{2}\left(X_{b}\right)
$$

This clearly implies theorem 3.2.

\section{SOME COROLLARIES}

Corollary 4.1. Let $S$ be a general $K 3$ surface of genus 5 , and let $X=S^{[2]}$ be the Hilbert square. Let $A \subset X$ be a general fibre of the Lagrangian fibration $\phi: X \rightarrow \mathbb{P}^{2}$ of proposition 2.25. Let $b \in A^{4}(X)$ be a $0-$ cycle of the form

$$
b=A \cdot a+p \quad \in A^{4}(X),
$$

where $a \in A^{2}(X)$ and $p$ is a sum of intersections of divisors and Chern classes of $X$. Then $b$ is rationally trivial if and only if $b$ is of degree 0.

Proof. The first thing to remark is that the Lagrangian surface $A$ is in $A_{(0)}^{2}(X)$. This follows from theorem 3.1, but it is far easier to note that a point $p \in \mathbb{P}^{2}$ is an intersection of two divisors, and so $A=\phi^{-1}(p)$ is an intersection of two divisors.

Next, one observes that the rational equivalence class of the surface $A \subset X$ exists relatively (this is because the Lagrangian fibration of proposition 2.25 exists relatively). Thus, one can apply theorem 3.2 to conclude that

$$
\cdot A: \quad A_{h o m}^{2}(X) \rightarrow A^{4}(X)
$$

is the zero map. This implies that $A \cdot a$ is in $A_{(0)}^{4}(X)$, and so also $b$ is in $A_{(0)}^{4}(X)$. Since $A_{(0)}^{4}(X) \cong \mathbb{Q}$, this proves the corollary.

Corollary 4.2. Let $g \in\{2,3,4,5,6,8\}$, and let $\mathcal{S} \rightarrow B$ be the universal family of genus $g K 3$ surfaces. Let $\mathcal{C} \subset \mathcal{S}$ be a "relative curve" (i.e., a divisor flat over $B$ ), and let $C_{b} \subset S_{b}$ denote the restriction $\left.\mathcal{C}\right|_{S_{b}}$. Let $X$ be the Hilbert scheme $X=\left(S_{b}\right)^{[2]}$ for any $b \in B$, and let $Y \subset X$ be the Lagrangian surface $\left(C_{b}\right)^{(2)}$. Then

$$
\begin{aligned}
& Y \in A_{(0)}^{2}(X), \\
& \cdot Y=0: \quad A_{\text {hom }}^{2}(X) \rightarrow A^{4}(X) .
\end{aligned}
$$

Proof. This is immediate from theorem 3.1.

Remark 4.3. In the set-up of corollary 4.2, it may be expected that $C_{b} \subset S_{b}$ is a constant cycle curve (this is Huybrechts' guess, cf. remark 3.3). This would imply that $Y \subset X$ is a constant cycle surface, which would nicely explain the behaviour of $Y$ exhibited in corollary 4.2. 
Corollary 4.4. Let $S \subset \mathbb{P}^{3}(\mathbb{C})$ be a smooth quartic, and let $X=S^{[2]}$ be the Hilbert scheme. Let $T \subset X$ be the surface of bitangents. Then

$$
\begin{aligned}
& T \in A_{(0)}^{2}(X), \\
& \cdot T=0: \quad A_{h o m}^{2}(X) \rightarrow A^{4}(X) .
\end{aligned}
$$

Proof. The surface $T \subset X$ exists relatively, and so this follows from theorem 3.1.

Remark 4.5. When $S \subset \mathbb{P}^{3}(\mathbb{C})$ is a generic quartic, there is Beauville's anti-symplectic involution $\iota: X \rightarrow X$ [1]. The fixed locus of $\iota$ is the surface of bitangents $T$ [6, Example 3.5]. The first part of corollary 4.4 thus follows from the more general statement that

$$
A^{2}(X)^{\iota} \subset A_{(0)}^{2}(X),
$$

which is proven in [25] (cf. also [12, Corollary 1.8]).

As for the second statement of corollary 4.4, it is perhaps possible to prove the stronger statement that $T \subset X$ is a constant cycle surface. (Since ८ acts as minus the identity on $A_{(2)}^{4}(X)$ [25], we know that

$$
\operatorname{Im}\left(A_{0}(T) \rightarrow A^{4}(X)\right) \cap A_{(2)}^{4}(X)=0 .
$$

To prove $T$ is a constant cycle surface, it only remains to prove that

$$
\operatorname{Im}\left(A_{0}(T) \rightarrow A^{4}(X)\right) \cap A_{(4)}^{4}(X) \stackrel{? ?}{=} 0 .
$$

I don't know how to do this.)

\section{FANO VARIETIES OF CUBIC FOURFOLDS}

In this final section, we prove a "generalized Franchetta conjecture" type of statement for the Fano variety of lines on a cubic fourfold containing two planes. The argument is similar to that of theorem 3.1.

Theorem 5.1. Let $\mathcal{Y} \rightarrow B$ be the universal family of smooth cubic fourfolds containing two disjoint planes, and let $\mathcal{X} \rightarrow B$ be the family of associated Fano varieties of lines ( $c f$. notation 2.29). Let $\Gamma \subset \mathcal{X}$ be a codimension 2 subvariety. The restriction $\left.\Gamma\right|_{X_{b}} \in A^{2}\left(X_{b}\right)$ is in $A_{(0)}^{2}\left(X_{b}\right)$ for all $b \in B$.

Proof. Let us consider the relative correspondence

$$
\Gamma_{0}:=\left(\Pi_{2}^{\mathcal{X}}\right)_{*}(\Gamma) \quad \in A^{2}(\mathcal{X}),
$$

where $\Pi_{2}^{\mathcal{X}}$ is the relative $\mathrm{CK}$ projector as in proposition 2.16. We know that there is fibrewise homological vanishing

$$
\left.\Gamma_{0}\right|_{X_{b}}=0 \quad \text { in } H^{4}\left(X_{b}\right) \quad \forall b \in B .
$$

What we need to prove (in order to have $\Gamma \in A_{(0)}^{2}\left(X_{b}\right)$ ) is that there is a fibrewise rational equivalence

$$
\left.\Gamma_{0}\right|_{X_{b}} \stackrel{? ?}{=} 0 \quad \text { in } A^{2}\left(X_{b}\right) \quad \forall b \in B
$$


To obtain this, we move things to the universal family of (squares of) related $K 3$ surfaces. That is, we first consider the relative correspondence

$$
\Gamma_{1}:=\left(\Psi_{1}\right)_{*}\left(\Gamma_{0}\right) \quad \in A^{4}\left(\mathcal{Y} \times_{B} \mathcal{Y}\right) \oplus \bigoplus A^{3}(\mathcal{Y})
$$

where $\Psi_{1} \in A^{6}\left(\mathcal{X} \times_{B}\left(\mathcal{Y} \times_{B} \mathcal{Y}\right)\right) \oplus \bigoplus A^{*}\left(\mathcal{X} \times_{B} \mathcal{Y}\right)$ is as in corollary 2.28. Let us write

$$
\Gamma_{1}=\Gamma_{1}^{0}+\Gamma_{1}^{1}+\cdots+\Gamma_{1}^{m},
$$

where $\Gamma_{1}^{0}$ is in $A^{4}\left(\mathcal{Y} \times{ }_{B} \mathcal{Y}\right)$ and $\Gamma_{1}^{i}$ is in $A^{3}(\mathcal{Y})$ for $i=1, \ldots, m$.

Next, we consider the relative correspondence

$$
\Gamma_{2}:=\left(\Psi_{3}\right)_{*}\left(\Gamma_{1}^{0}\right)+\sum_{i=1}^{m}\left(\Psi_{2}\right)_{*}\left(\Gamma_{1}^{i}\right) \quad \in A^{2}\left(\mathcal{S} \times_{B} \mathcal{S}\right) \oplus \bigoplus_{j=1}^{m+2} A^{2}(\mathcal{S}),
$$

where $\Psi_{2}$ and $\Psi_{3}$ are as in lemma 2.30 resp. lemma 2.31. Let us split this correspondence into two parts, writing

$$
\Gamma_{2}=\Gamma_{2}^{\prime}+\Gamma_{2}^{\prime \prime}
$$

with

$$
\Gamma_{2}^{\prime} \in A^{2}\left(\mathcal{S} \times{ }_{B} \mathcal{S}\right), \quad \Gamma_{2}^{\prime \prime} \in \bigoplus A^{2}(\mathcal{S})
$$

Property (17) implies there is a fibrewise homological vanishing

$$
\left.\left(\Gamma_{2}^{\prime}+\Gamma_{2}^{\prime \prime}\right)\right|_{S_{b} \times S_{b}}=0 \quad \text { in } H^{4}\left(S_{b} \times S_{b}\right) \oplus \bigoplus H^{4}\left(S_{b}\right) \quad \forall b \in B .
$$

The family $\mathcal{S} \rightarrow B$ fulfills the conditions of proposition 2.22 (with $M=\mathbb{P}^{2} \times \mathbb{P}^{2}$ and $L_{1}=$ $\mathcal{O}_{M}(1,2)$ and $\left.L_{2}=\mathcal{O}_{M}(2,1)\right)$. Applying proposition 2.22 to $\Gamma_{2}^{\prime}$, we find that there exists $\delta \in$ $A^{2}(M \times M)$ such that

$$
\left.\Gamma_{2}^{\prime}\right|_{S_{b} \times S_{b}}=\left.\delta\right|_{S_{b} \times S_{b}} \text { in } A^{2}\left(S_{b} \times S_{b}\right) \quad \forall b \in B .
$$

Combined with (19), this implies in particular that

$$
\left.\delta\right|_{S_{b} \times S_{b}} \in A_{\text {hom }}^{2}\left(S_{b} \times S_{b}\right) \quad \forall b \in B .
$$

But $A^{*}(M \times M)=A^{*}(M) \otimes A^{*}(M)$ is generated by divisors, and so the restriction $\left.\delta\right|_{S_{b} \times S_{b}}$ is in the subring generated by divisors. It is known this subring injects into cohomology [42], and so

$$
\left.\delta\right|_{S_{b} \times S_{b}}=0 \quad \text { in } A^{2}\left(S_{b} \times S_{b}\right) \quad \forall b \in B .
$$

It follows that also

$$
\left.\Gamma_{2}^{\prime}\right|_{S_{b} \times S_{b}}=0 \quad \text { in } A^{2}\left(S_{b} \times S_{b}\right) \quad \forall b \in B .
$$

Next, let us consider the part $\Gamma_{2}^{\prime \prime}$. We have seen (equality (19)) that this cycle is fibrewise homologically trivial:

$$
\left.\Gamma_{2}^{\prime \prime}\right|_{\left(S_{b}\right) \oplus m+2}=0 \quad \text { in } \bigoplus_{j=1}^{m+2} H^{4}(\mathcal{S}) \quad \forall b \in B
$$


It follows from proposition 2.33 that there is a rational equivalence

$$
\left.\Gamma_{2}^{\prime \prime}\right|_{\left(S_{b}\right) \oplus m+2}=0 \quad \text { in } \bigoplus_{j=1}^{m+2} A^{2}(\mathcal{S}) \quad \forall b \in B .
$$

Combining (20) and (21), we find that

$$
\left.\Gamma_{2}\right|_{\left(S_{b} \times S_{b}\right) \cup\left(S_{b}\right)^{\oplus m+2}}=0 \quad \text { in } A^{2}\left(S_{b} \times S_{b}\right) \oplus \bigoplus_{j=1}^{m+2} A^{2}\left(S_{b}\right) .
$$

Let us now get back to the Fano varieties $X_{b}$. We know that

$$
\left(\Psi_{1}\right): \quad A_{\text {hom }}^{2}\left(X_{b}\right) \rightarrow A_{\text {hom }}^{4}\left(Y_{b} \times Y_{b}\right) \oplus \bigoplus_{i=1}^{m} A_{h o m}^{3}\left(Y_{b}\right)
$$

is injective (corollary 2.28). Likewise, we know that

$$
\begin{aligned}
\left(\Psi_{2}\right)_{*}: \quad A_{\text {hom }}^{3}\left(Y_{b}\right) & \rightarrow A^{2}\left(S_{b}\right), \\
\left(\Psi_{3}\right)_{*}: \quad A_{\text {hom }}^{4}\left(Y_{b} \times Y_{b}\right) & \rightarrow A^{2}\left(S_{b} \times S_{b}\right) \oplus A^{2}\left(S_{b}\right)^{\oplus 2}
\end{aligned}
$$

are injective, at least for general $b \in B$ (lemmas 2.30 and 2.31).

Looking at the definition of $\Gamma_{2}$, the vanishing (22) thus implies that

$$
\left.\Gamma_{0}\right|_{X_{b}}=0 \text { in } A^{2}\left(X_{b}\right) \text { for very general } b \in B .
$$

In view of [45, Lemma 3.2], this implies that actually

$$
\left.\Gamma_{0}\right|_{X_{b}}=0 \text { in } A^{2}\left(X_{b}\right) \quad \forall b \in B
$$

as desired, proving equality (18) and hence theorem 5.1.

Remark 5.2. One can also prove the analogue of theorem 3.2 for Fano varieties $X_{b}$ as in theorem 5.1. The details will appear elsewhere.

Acknowledgements. Thanks to the referee for pertinent and helpful comments. Thanks to all the members of Het Lieve Team, in particular Kai-kun, Len-kun \& Yoyo-chan.

\section{REFERENCES}

[1] A. Beauville, Some remarks on Kähler manifolds with $c_{1}=0$, in: Classification of algebraic and analytic manifolds (Katata, 1982), Birkhäuser Boston, Boston 1983,

[2] A. Beauville, Variétés Kähleriennes dont la première classe de Chern est nulle, J. Differential Geom. 18 no. 4 (1983), 755-782,

[3] A. Beauville and R. Donagi, La variété des droites d'une hypersurface cubique de dimension 4, C. R. Acad. Sci. Paris Sér. I Math. 301 no. 14 (1985), 703-706,

[4] A. Beauville and C. Voisin, On the Chow ring of a K3 surface, J. Alg. Geom. 13 (2004), 417—426,

[5] A. Beauville, On the splitting of the Bloch-Beilinson filtration, in: Algebraic cycles and motives (J. Nagel et al., eds.), London Math. Soc. Lecture Notes 344, Cambridge University Press 2007, 
[6] A. Beauville, Antisymplectic involutions of holomorphic symplectic manifolds, Journal of Topology 4 no. 2 (2011), 300-304,

[7] S. Bloch, Lectures on algebraic cycles, Duke Univ. Press Durham 1980,

[8] A. Ferretti, The Chow ring of double EPW sextics, Algebra and Number Theory 6(3) (2009),

[9] B. Fu, Abelian fibrations on $S^{[n]}$, C. R. Math. Acad. Sci. Paris 337 no. 9 (2003), 593-596,

[10] L. Fu, Beauville-Voisin conjecture for generalized Kummer varieties, Int. Math. Res. Notices 12 (2015), 3878-3898, 2014,

[11] L. Fu, On the action of symplectic automorphisms on the $C H_{0}$-groups of some hyper-Kähler fourfolds, Math. Z. 280 (2015), 307-334,

[12] L. Fu, R. Laterveer and Ch. Vial, The generalized Franchetta conjecture for some hyper-Kähler varieties (with an appendix joint with M. Shen), arXiv:1708.02919v2, to appear in Journal de Math. Pures et Appliquées,

[13] L. Fu, Z. Tian and C. Vial, Motivic hyperkähler resolution conjecture: I. Generalized Kummer varieties, to appear in Geom. Topol.,

[14] W. Fulton, Intersection theory, Springer-Verlag Ergebnisse der Mathematik, Berlin Heidelberg New York Tokyo 1984,

[15] S. Galkin and E. Shinder, The Fano variety of lines and rationality problem for a cubic hypersurface, arXiv:1405.5154,

[16] B. Hassett, Some rational cubic fourfolds, J. Alg. Geom. 8(1) (1999), 103-114,

[17] B. Hassett, Cubic fourfolds, $K 3$ surfaces and rationality questions, in: Rationality problems in algebraic geometry (R. Pardini et al., eds.), Springer Lecture Notes in Mathematics, Springer 2016,

[18] B. Hassett and Y. Tschinkel, Abelian fibrations and rational points on symmetric products, International Journal of Mathematics 11(9) (2000), 1163-1176,

[19] D. Huybrechts, Curves and cycles on $K 3$ surfaces, Algebraic Geometry 1 (2014), 69-106,

[20] A. Iliev and K. Ranestad, The abelian fibration on the Hilbert cube of a $K 3$ surface of genus 9, International Journal of Mathematics 18(1) (2007),

[21] U. Jannsen, Motivic sheaves and filtrations on Chow groups, in: Motives (U. Jannsen et al., eds.), Proceedings of Symposia in Pure Mathematics Vol. 55 (1994), Part 1,

[22] U. Jannsen, On finite-dimensional motives and Murre's conjecture, in: Algebraic cycles and motives (J. Nagel et al., eds.), Cambridge University Press, Cambridge 2007,

[23] S. Kimura, Chow groups are finite dimensional, in some sense, Math. Ann. 331 (2005), 173-201,

[24] R. Laterveer, A remark on the motive of the Fano variety of lines of a cubic, Ann. Math. Québec 41 no. 1 (2017), 141-154,

[25] R. Laterveer, Bloch's conjecture for some hyperkähler fourfolds, to appear in Pure and Applied Math. Quarterly,

[26] H. Lin, On the Chow groups of zero-cycles of a generalized Kummer variety, Advances in Mathematics 298 (2016), 448-472,

[27] H. Lin, Lagrangian constant cycle subvarieties in Lagrangian fibrations, to appear in Int. Math. Res. Notices,

[28] S. Mukai, Symplectic structure of the moduli space of sheaves on an abelian or $K 3$ surface., Invent. Math. 77 no. 1 (1984), 101-116,

[29] S. Mukai, Curves, $K 3$ surfaces and Fano 3 -folds of genus $\leq 10$, in: Algebraic geometry and commutative algebra (H. Hijikata et al., eds.), Kinokuniya Tokyo 1988,

[30] J. Murre, On a conjectural filtration on the Chow groups of an algebraic variety, parts I and II, Indag. Math. 4 (1993), 177-201,

[31] J. Murre, J. Nagel and C. Peters, Lectures on the theory of pure motives, Amer. Math. Soc. University Lecture Series 61, Providence 2013,

[32] K. O'Grady, Moduli of sheaves and the Chow group of $K 3$ surfaces, Journal de Math. Pures et Appliquées 100 no. 5 (2013), 701-718, 
[33] N. Pavic, J. Shen and Q. Yin, On O'Grady's generalized Franchetta conjecture, Int. Math. Res. Notices 2016 (2016), 1-13,

[34] U. Rieß, On the Chow ring of birational irreducible symplectic varieties, Manuscripta Math. 145 (2014), $473-501$,

[35] U. Rieß, On Beauville's conjectural weak splitting property, Int. Math. Res. Notices 2016 no. 20 (2016), 6133-6150,

[36] J. Sawon, Lagrangian fibrations on Hilbert schemes of points on $K 3$ surfaces, Journal of Alg. Geom. 16(3) (2007), 477-497,

[37] T. Scholl, Classical motives, in: Motives (U. Jannsen et alii, eds.), Proceedings of Symposia in Pure Mathematics Vol. 55 (1994), Part 1,

[38] M. Shen and C. Vial, The Fourier transform for certain hyperKähler fourfolds, Memoirs of the AMS 240 (2016), no.1139,

[39] M. Shen and C. Vial, The motive of the Hilbert cube $X^{[3]}$, Forum Math. Sigma 4 (2016),

[40] C. Vial, Remarks on motives of abelian type, Tohoku Math. J. 69 (2017), 195-220,

[41] C. Vial, On the motive of some hyperkähler varieties, J. für Reine u. Angew. Math. 725 (2017), 235-247,

[42] C. Voisin, On the Chow ring of certain algebraic hyperkähler manifolds, Pure Appl. Math. Q. 4 no. 3 part 2 (2008), 613-649,

[43] C. Voisin, The generalized Hodge and Bloch conjectures are equivalent for general complete intersections, Ann. Sci. Ecole Norm. Sup. 46, fascicule 3 (2013), 449-475,

[44] C. Voisin, The generalized Hodge and Bloch conjectures are equivalent for general complete intersections, II, J. Math. Sci. Univ. Tokyo 22 (2015), 491-517,

[45] C. Voisin, Chow Rings, Decomposition of the Diagonal, and the Topology of Families, Princeton University Press, Princeton and Oxford, 2014,

[46] C. Voisin, Hodge structures, Coniveau and Algebraic Cycles, in: "The Legacy of Bernhard Riemann After One Hundred and Fifty Years", ALM35, Higher Education Press and International Press, Beijing Boston 2016,

[47] C. Voisin, Remarks and questions on coisotropic subvarieties and 0-cycles of hyper-Kähler varieties, in: K3 Surfaces and Their Moduli, Proceedings of the Schiermonnikoog conference 2014 (C. Faber et al., eds.), Progress in Maths 315, Birkhäuser 2016,

[48] C. Voisin, On the universal $C H_{0}$ group of cubic hypersurfaces, Journal Eur. Math. Soc. 19 no. 6 (2017), $1619-1653$.

Institut de Recherche Mathématique Avancée, CNRS - Université de Strasbourg, 7 Rue RenÉ Descartes, 67084 STRASBourg CEDEX, FRANCE.

E-mail address: robert. laterveer@math.unistra.fr 